\title{
Theta oscillations in the prefrontal-hippocampal circuit do not couple to respiration-
}

\section{related oscillations}

Sunandha Srikanth ${ }^{1}$, Dylan Le ${ }^{1}$, Yudi Hu${ }^{1}$, Jill K. Leutgeb ${ }^{1, *}$, Stefan Leutgeb ${ }^{1,2, *}$

${ }^{1}$ Neurobiology Section, Division of Biological Sciences, University of California, San Diego, La

$$
\text { Jolla, CA 92093, USA }
$$

${ }^{2}$ Kavli Institute for Brain and Mind, University of California, San Diego, La Jolla, CA 92093, USA

\footnotetext{
* Correspondence should be addressed to: sleutgeb@ucsd.edu, jleutgeb@ucsd.edu
} 


\section{ABSTRACT}

2 Oscillatory activity is thought to coordinate neural computations across brain regions, and theta

3 oscillations are critical for learning and memory. Because the frequency of respiratory-related

4 oscillations (RROs) in rodents can overlap with the frequency of theta in the prefrontal cortex

5 (PFC) and the hippocampus, we asked whether odor-cued working memory may be supported by

6 coupling between these two oscillations. We first confirmed that RROs are propagated to the

7 hippocampus and PFC and that RRO frequency overlaps with canonical theta frequency. However,

8 we found low coherence between RROs and local theta oscillations in the hippocampus-PFC

9 network when the two types of oscillations overlapped in frequency. This effect was observed

10 during all behavioral phases including during movement and while odors were actively sampled

11 when stationary. Despite the similarity in frequency, RROs and theta oscillations therefore appear

12 to be limited to supporting computation in distinct networks, which suggests that sustained long-

13 range coordination between oscillation patterns that depend on separate pacemakers is not

14 necessary to support at least one type of working memory. 


\section{INTRODUCTION}

16 Brain oscillations are thought to coordinate neural computations across cortical and sub-cortical

17 brain regions by synchronizing network activity (Buzsáki \& Draguhn, 2004). In brain circuits that

18 support memory function, such coordination is most prominent in the theta frequency range (6-12

$19 \mathrm{~Hz}$ ). Theta oscillations are not only prominent in the hippocampus, but matching oscillations can

20 also be observed in directly and indirectly connected brain regions (Backus et al., 2016; Buzsáki,

21 2002; Colgin, 2011). For example, local field potentials (LFP) are highly coherent between

22 hippocampus and medial prefrontal cortex (mPFC), and neuronal firing patterns of many prefrontal

23 neurons are phase-locked to the hippocampal theta rhythm (Hyman et al., 2005; Jones \& Wilson,

24 2005; Siapas et al., 2005; Zielinski et al., 2019). Since oscillations within a network correspond to

25 cyclic fluctuations in excitability, such synchronized oscillations across brain regions allow for

26 windows of peak excitability that enable efficient communication between the brain regions (Fries,

27 2005). Accordingly, the accuracy of spatial coding in hippocampus and mPFC has been reported

28 to be coupled on a cycle-by-cycle basis (Zielinski et al., 2019). Furthermore, prefrontal-

29 hippocampal oscillatory strength correlates with performance in spatial working memory tasks in

30 rodents (Benchenane et al., 2010; Jones \& Wilson, 2005; Zielinski et al., 2019), which suggests

31 that oscillatory coupling supports memory function and raises the question whether an even

32 broader network is dynamically synchronized during task performance.

34 Along with the canonical theta oscillations that are most prominent in the hippocampus,

35 oscillations that encompass an overlapping frequency range $(3-12 \mathrm{~Hz})$ and are related to the

36 respiration rhythm have also been described (Lockmann et al., 2016; Nguyen Chi et al., 2016;

37 Rojas-Líbano et al., 2014; Yanovsky et al., 2014). Respiration is paced by brainstem breathing 
centers (Feldman et al., 2013), and the nasal airflow that is generated by breathing then activates olfactory sensory neurons in the nasal epithelium during each breathing cycle (Wu et al., 2017). This mechanism entrains local oscillatory activity in the olfactory bulb (OB), and the respiratory rhythm and $\mathrm{OB}$ oscillations are thus tightly coupled. In particular, a causal role of nasal airflow

42 for olfactory oscillations has been established by the finding that the entrainment of OB network

43 activity is diminished when nasal airflow is restricted by means of naris occlusion or tracheal

44 breathing (Onoda \& Mori, 1980; Phillips et al., 2012).

Respiration-entrained activity of OB neurons is transmitted to olfactory-associated cortical areas such as the piriform cortex (Fontanini et al., 2003) and the barrel cortex (Ito et al., 2014), but also to more indirectly connected subcortical and cortical regions across the brain, including the medial prefrontal cortex (mPFC) (Biskamp et al., 2017) and the hippocampus (Lockmann et al., 2016; Nguyen Chi et al., 2016; Tort et al., 2018; Yanovsky et al., 2014). Throughout these brain regions,

51 respiration-related oscillations (RROs) can be distinguished from other types of oscillations by

52 confirming the coupling to either the respiration rhythm and/or olfactory bulb oscillations.

53 Consistent with the definition of RROs as respiration or OB-oscillation related, these oscillatory

54 patterns in the mPFC, barrel cortex and the hippocampus are disrupted when manipulating signals

55 from the OB through bulbectomies and olfactory epithelial ablation or when disturbing nasal 56 airflow through tracheotomies and naris occlusions (Biskamp et al., 2017; Ito et al., 2014; Moberly 57 et al., 2018; Nguyen Chi et al., 2016; Yanovsky et al., 2014).

59 Because the overlap in frequency between RROs and canonical theta can be confounding for 60 separately analyzing these oscillation patterns, characterization of RROs has mostly focused on 
61 periods when RROs differ in frequency from theta oscillations during running, immobility and

62 anesthesia (Nguyen Chi et al., 2016; Yanovsky et al., 2014). In these analyses, oscillations at the

63 respiratory frequency have a different depth profile than theta oscillations in the hippocampus,

64 which supports the notion that RROs are separate oscillations that co-occur with theta oscillations

65 in the hippocampus. In contrast, there is also evidence that olfactory oscillations and hippocampal

66 theta oscillations become coherent during periods of sniffing in odor learning and discrimination

67 tasks (Kay, 2005; Macrides et al., 1982). These latter studies suggest that the coherence between

68 hippocampal and olfactory networks mediates sensorimotor integration in the hippocampus. A

69 possible source for the conflicting reports on the coupling of respiratory oscillations and

70 hippocampal oscillations is that these reports have not considered the existence of two types of

71 theta oscillations in the hippocampus - movement-related theta oscillations and sensory-evoked

72 theta oscillations (Kramis et al., 1975; Vanderwolf, 1969). We therefore investigated whether

73 coupling between RROs and theta oscillation may differ depending on the behavioral state during

74 which theta is generated. Furthermore, we reasoned that coupling between the respiratory rhythm

75 and hippocampal canonical theta may be required when olfactory cues are relevant for memory

76 performance and performed recordings in an odor-cued hippocampus-dependent working memory

77 task. To be able to identify RROs throughout the behavior, we recorded OB oscillations

78 simultaneously with hippocampal oscillations. Furthermore, we also simultaneously recorded

79 from mPFC to examine whether the convergence of RROs and hippocampus-coupled theta in

80 mPFC would allow for dynamic coupling between these two types of oscillations, which could in

81 turn serve as a conduit for coordinating memory and sensory processing in the prefrontal-

82 hippocampal circuit. 


\section{RESULTS}

85 To investigate the coupling between the respiration-coupled oscillations in the OB and theta oscillations in the mPFC and hippocampus, we simultaneously recorded LFP signals across these

87 brain regions. Because ventral hippocampus (vHC) is connected more strongly to mPFC than dorsal hippocampus (dHC) (Hoover \& Vertes, 2007), we placed separate recording electrodes in the $\mathrm{dHC}$ and the vHC. Within mPFC, we focused on the prelimbic, infralimbic, and anterior

91 as theta oscillations have been detected in all of these regions in previous studies (Tort et al., 2018).

A
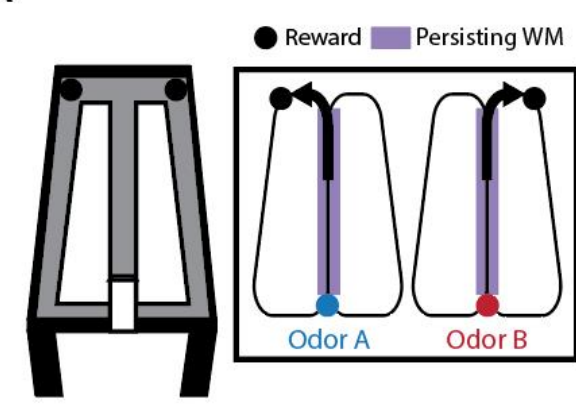

C

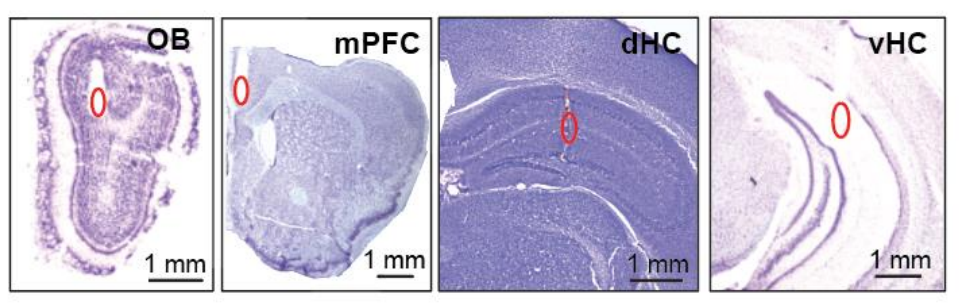

B

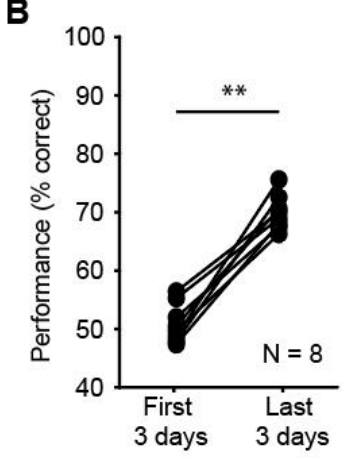

Figure 1. Mice performed an odor-cued working memory task with high accuracy. A. Schematic of the odor-cued working memory task. Mice were trained to sniff one of two pseudorandomly delivered odors at an odor port at the bottom of the stem arm and make a turn at the top of the stem arm based on the odor they sampled. The relation between odor identity and turn direction remained consistent for each mouse. A food reward was provided the reward zones for correct choices, and mice returned to the odor port on the side arms. B. Performance increased between the first three days and the last three days of behavioral testing $(n=8$ mice, $\mathrm{Z}=2.45, \mathrm{p}=0.007$, one-tailed Wilcoxon signed-rank test) $\mathrm{C}$. Example recording electrode locations in the olfactory bulb (OB), medial prefrontal cortex (mPFC), dorsal hippocampus (dHC) and ventral hippocampus (vHC). Recording locations are highlighted (red ovals) in cresyl-violet stained brain slices. 
93 To be able to examine oscillations across a range of behavioral states, we trained mice in an odor-

94 cued working memory task (Figure 1A) in which we could observe movement-related

95 hippocampal theta while mice ran between an odor port and reward locations and sensory-related

96 theta when mice sampled odors while they remained stationary at the odor port. Briefly, mice $(n=$

97 8) were trained to run on a figure- 8 maze in which an odor port was placed at one end of the stem

98 arm where one of two odors (isoamyl acetate or ethyl acetate) was delivered in a pseudorandom

99 fashion. Mice were trained to sample the odor by poking and holding their nose in the odor port

100 for at least one second. They then had to retain information about the odor identity while running

101 to the opposite end of the stem arm to make their choice to turn left or right. Correct choices were

102 rewarded with a single chocolate sprinkle. As expected, initial performance was at chance level ( $n$

$103=8$ mice, $\mathrm{Z}=0.49, \mathrm{p}=0.31$, one-tailed Wilcoxon signed-rank test). Using a criterion of $65 \%$

104 correct during at least 2 of 3 consecutive days, mice learned the task within $15 \pm 5$ days.

105 Accordingly, performance during the last three days of testing was better than during the first three

106 days (median: $69.5 \%$ vs $50.2 \%$ correct, $n=8$ mice, $Z=2.45, p=0.007$, one-tailed Wilcoxon

107 signed-rank test; Figure 1B). All analyses of electrophysiological data were performed on data

108 from the last three testing days for each animal. Recording sites in the OB, mPFC, dHC and vHC

109 were confirmed in histological material (Figure 1C). Since histological confirmation of electrode

110 locations was not successful in one animal, we included only 7 of 8 animals for all LFP analysis.

\section{Predominant $\mathrm{OB}$ frequencies ranged from 3-12 $\mathrm{Hz}$ in all task phases}

113 The task was parsed into four task phases with distinct behavioral patterns - return arm where

114 animals ran without a memory load, odor sampling when animals actively sampled an odor, stem

115 arm where animals ran after odor sampling and before making a choice, and reward arm where 
116 animals were rewarded for correct performance. Time periods when animals transitioned between

117 these phases were not considered. During the odor sampling period, the animals poked their noses

118 into the odor port and sampled the odor while holding the nose in the odor port and were thus

119 stationary. For meaningful comparisons with the stationary odor sampling periods, another period

120 with minor movements was selected by restricting analyses on the reward arm to periods with low

121 velocity (less than $5 \mathrm{~cm} / \mathrm{s}$ ). Conversely, we confirmed that running speeds on the return arms and

122 on the stem were high and matched (Figure 2A), which allowed us to compare two task phases

123 with corresponding movement patterns.

A
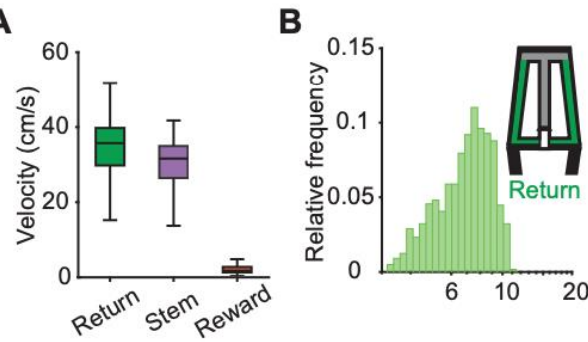

C
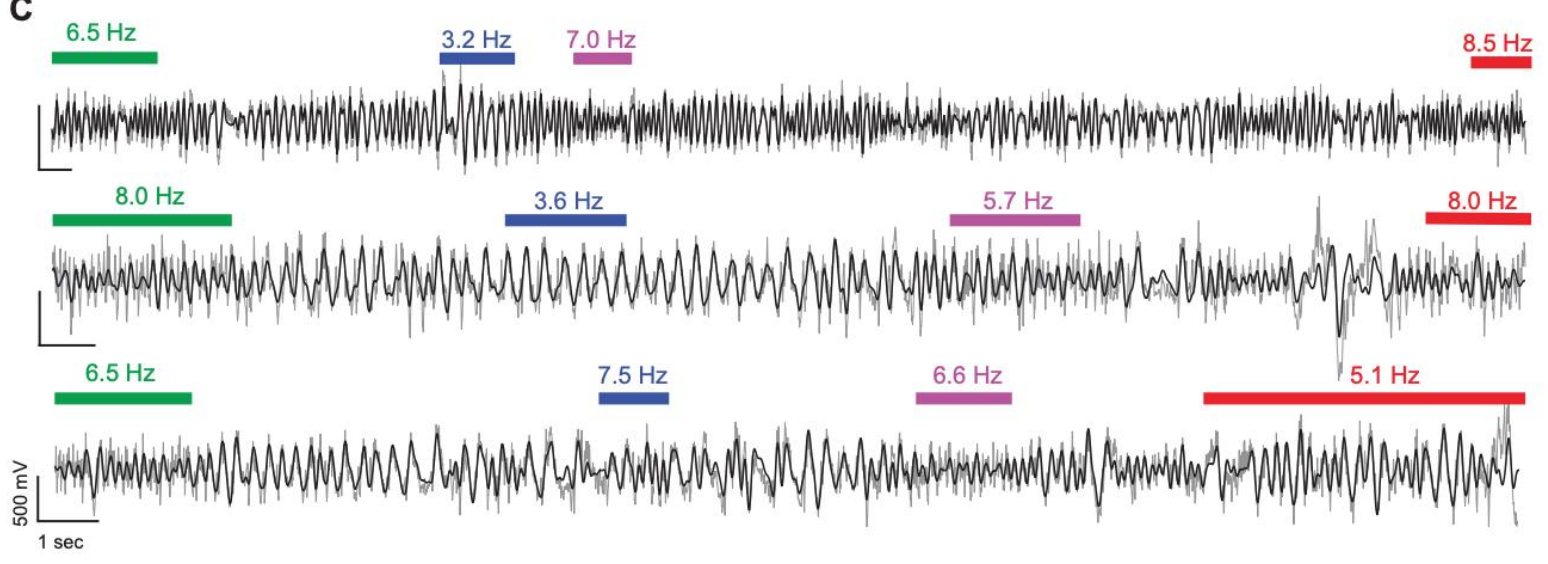

Figure 2. OB frequencies within each task phase ranged from 3-12 Hz. A. Velocity of the mice ( $n=7$ mice, 1207 trials) in each maze zone. Velocity in the odor port is not shown and was near zero while animals had to hold their nose in the port during odor sampling. In the box plots, the center line shows the median, and the bottom and top edges of the box represent the 25 th and 75th percentiles, respectively. The whiskers indicate the most extreme data points. B. The predominant $\mathrm{OB}$ frequency in each trial was calculated and the distribution of predominant $\mathrm{OB}$ frequencies across trials is plotted for each task phase. C. Example OB LFP traces (grey: raw traces, black: 3-12 Hz filtered traces). Each line is a trial, and colored bars indicate time periods when animals were in the respective task phase (green: return arm, blue: odor sampling, purple: stem arm and red: reward zone). Numbers on top of bars indicate the predominant OB frequency. Transition phases are without bars and were not analyzed. 
124 Although the velocity profiles ranged from immobility in the odor port to high running speeds on

125 the return arms (Figure 2A), the frequency distributions of OB oscillations across the four task

126 phases showed only minor differences $(n=1207$ trials, median \pm iqr in return: $7.02 \pm 2.77 \mathrm{~Hz}$; stem:

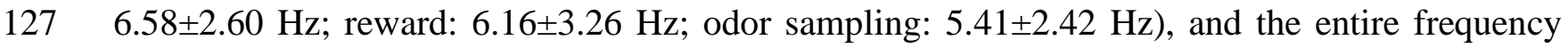

128 range from $3 \mathrm{~Hz}$ to $12 \mathrm{~Hz}$ was observed across trials for each of the phases (Figure 2B). Moreover,

129 within a trial, predominant OB frequencies varied across task phases (Figure 2C) with only weak

130 correlations among them (Spearman correlation coefficients <0.5) (Figure 2 - figure
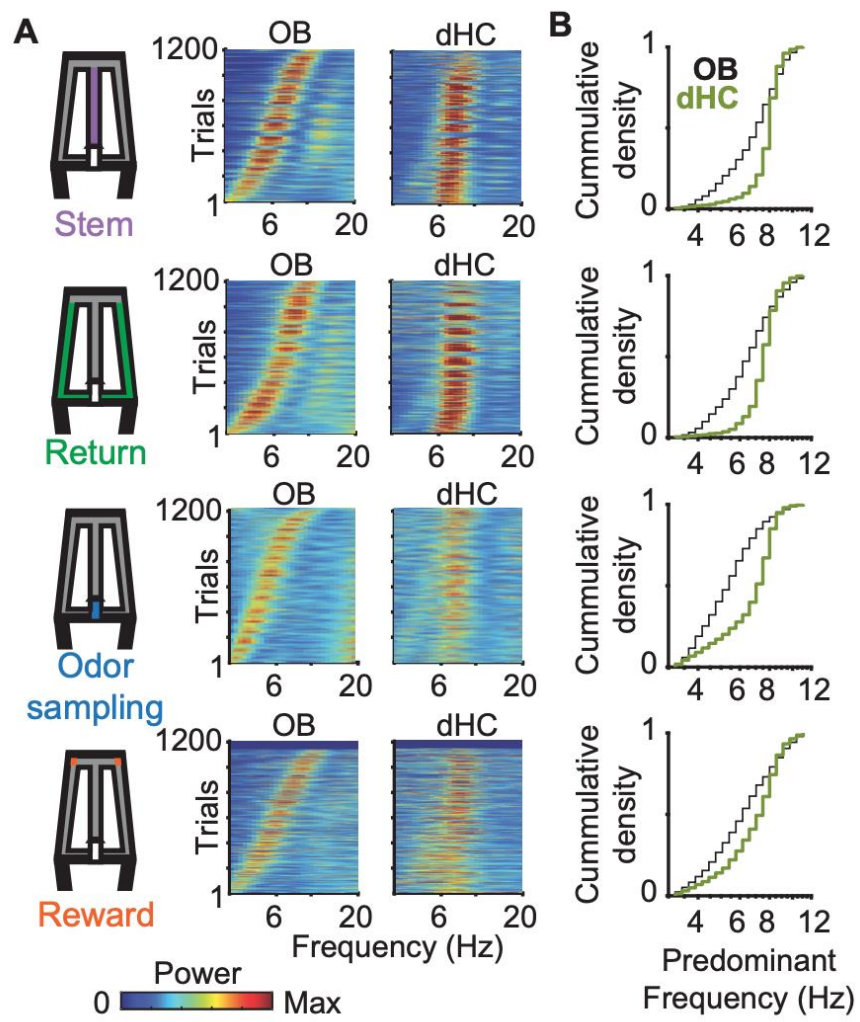

Figure 3. Across trials, predominant $\mathrm{OB}$ oscillation frequencies and $\mathrm{dHC}$ oscillation frequencies varied so that they were either overlapping or non-overlapping. A. Power spectra of $\mathrm{OB}$ and $\mathrm{dHC}$ oscillations are shown as color-coded plots, with each line corresponding to a trial. Trials are ordered by the OB peak oscillation frequency. B. Cumulative density functions of the predominant $\mathrm{OB}$ frequencies (black) and $\mathrm{dHC}$ frequencies (green) for the four task phases. The data for OB frequencies is replotted from Figure 2A for comparisons with $\mathrm{dHC}$ frequencies. Predominant $\mathrm{dHC}$ frequencies were concentrated in the range of 7-11 $\mathrm{Hz}$, while $\mathrm{OB}$ frequencies spanned the entire range of 3-12 Hz during all the task phases. Frequency distributions differed between brain regions in all task phases $(n=1207$ trials, Return: $\mathrm{p}=3.4 \mathrm{e}-56, \mathrm{KS}=0.32$; Stem: $\mathrm{p}=1.8 \mathrm{e}-70, \mathrm{KS}=0.36$; Odor sampling: $\mathrm{p}=7.1 \mathrm{e}-88, \mathrm{KS}$ $=0.41$; Reward: $\mathrm{p}=4.7 \mathrm{e}-21, \mathrm{KS}=0.20)$. 
131 supplement 1). OB oscillations in our analyzed frequency range have been firmly established as

132 being generated by respiration (Jessberger et al., 2016; Phillips et al., 2012; Rojas-Líbano et al.,

133 2014), and we thus refer to these oscillations as respiratory-related oscillations (RROs).

\section{RROs and canonical theta differed in their frequency distributions}

136 Because our task design included phases with running and immobility, it allowed us to assess the

137 coordination of RROs with theta across these task phases. As expected for movement-related theta,

138 high amplitude theta oscillations were observed during periods of running on the stem and return

139 arms (Figure 3A and Figure 3 - figure supplement 1A). Hippocampal theta oscillations were

140 also observed during odor sampling, and because mice were stationary while holding the nose in

141 the odor port, theta oscillations during this task phase can be considered sensory evoked (Figure

142 3A and Figure 3 - figure supplement 1B). Sensory-evoked theta oscillations were lower in

143 amplitude than movement-related theta oscillations (Figure 3A) but could nonetheless be clearly

144 detected in the $\mathrm{dHC}$ as distinct from RROs based on their frequency distribution (Figure 3B and

145 Figure 3 - figure supplement 1C). The predominant frequencies of the hippocampal oscillations

146 during odor sampling were as narrowly distributed as during movement, while RROs varied more

147 widely in frequency than the hippocampal oscillations (Figure 3B and Figure $\mathbf{3}$ - figure

148 supplement 1C and D). Hippocampal theta oscillations were also detected on reward arms, but

149 this period included bouts with low movement velocity $(<5 \mathrm{~cm} / \mathrm{s})$ such that the type of theta cannot

150 be as clearly classified as during other phases of behavior. Irrespective of behavior phase, the

151 distribution of predominant frequencies in OB therefore included the entire 3-12 Hz range while

152 canonical dHC theta was mostly concentrated in the 7-11 Hz range (Figure 3 - figure supplement

153 1C, Figure 3B). 
A

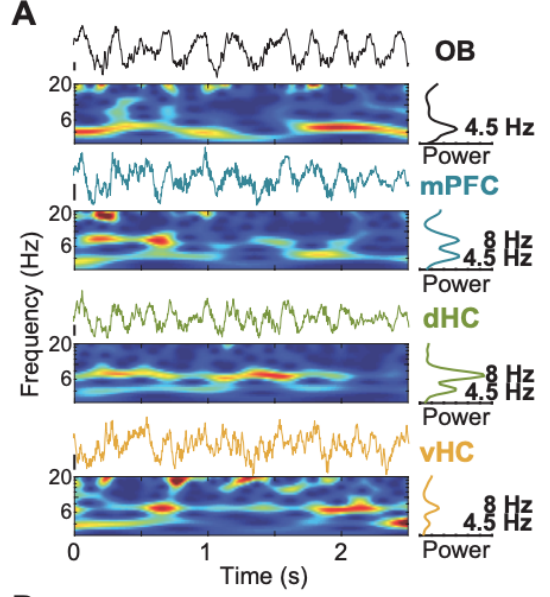

D

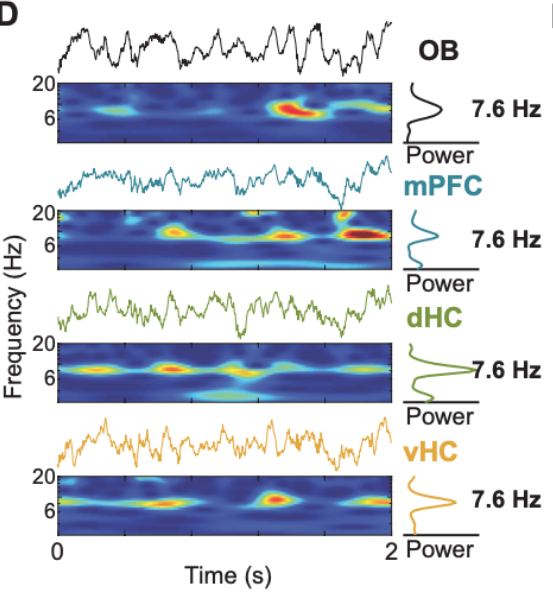

B

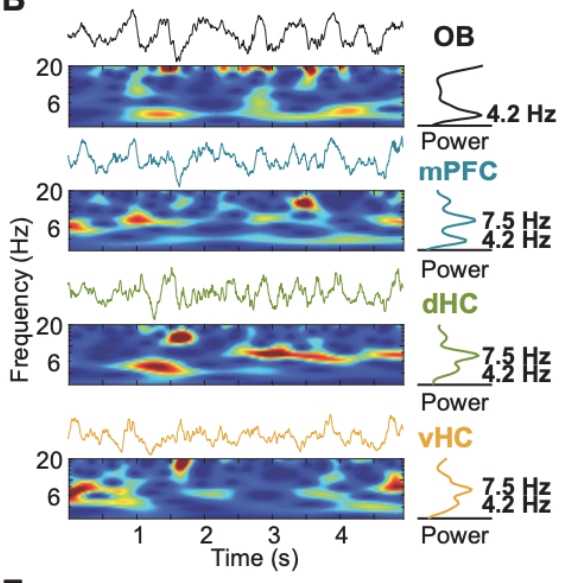

E

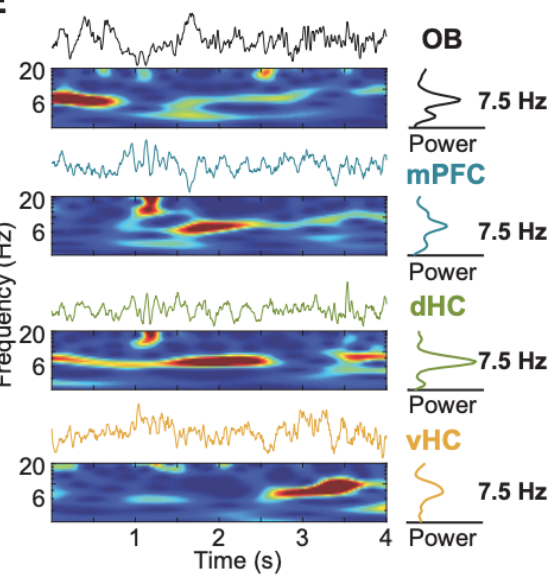

C

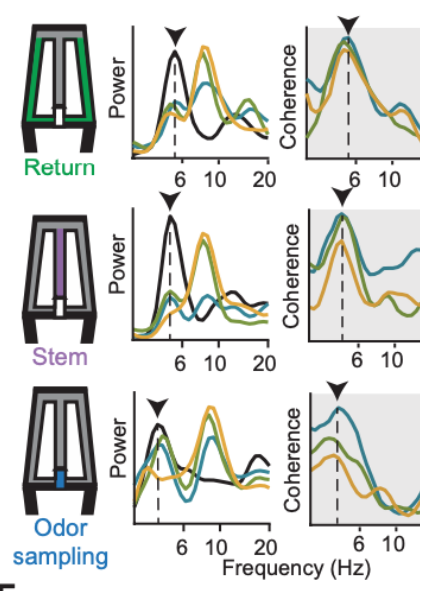

$\mathbf{F}$

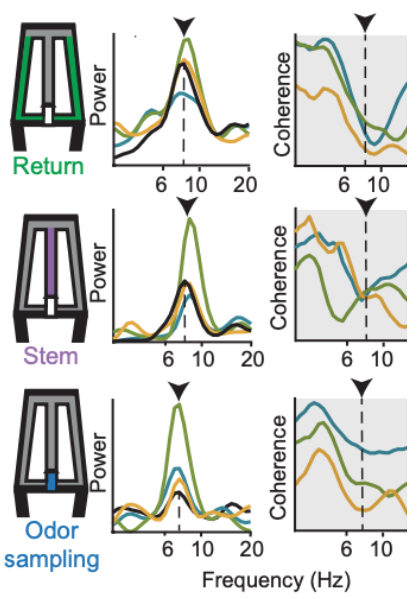

Figure 4. RROs were observed in the mPFC-dHC-vHC network in parallel with movement-related or sensory-evoked theta oscillations. A. Example raw traces and corresponding time-frequency spectrograms of simultaneously recorded LFP from the OB, $\mathrm{mPFC}, \mathrm{dHC}$ and $\mathrm{vHC}$ are shown for a period when the animal was running on the stem arm of the maze. In the example, theta frequency in the mPFC-dHC-vHC regions was nonoverlapping with the predominant $\mathrm{OB}$ frequency. B. Arranged as in A but for an example period when the mouse was stationary while actively sampling odor at the port. As in A, theta and $\mathrm{OB}$ frequencies were non-overlapping. C. Time averaged power spectra (left) and coherence spectra (right) are shown for three example periods within a trial and maze zone (return arm, stem arm and odor sampling period) when OB and canonical theta frequencies were non-overlapping. Dotted lines and arrows indicate the frequency of the predominant $\mathrm{OB}$ oscillation in the respective trials. OB-mPFC, OB-dHC and OB-vHC coherence is higher at the frequency matching the predominant $\mathrm{OB}$ frequency compared to the theta frequency. $\mathbf{D}, \mathbf{E}$. Arranged as in A and B, respectively, but for example periods when OB and canonical theta oscillations overlapped in frequency. F. Arranged as in $\mathrm{C}$ but for example periods (return arm, stem arm and odor sampling period) with overlapping $\mathrm{OB}$ and canonical theta frequencies. Despite the similar peak frequencies of both types of oscillations, OB-mPFC, OB-dHC and $\mathrm{OB}-\mathrm{vHC}$ coherence was low at the frequency matching the predominant $\mathrm{OB}$ frequency. Accordingly, canonical theta oscillations in the mPFC-dHC-vHC network did not couple to respiration-entrained oscillations. 
154 The wider frequency range for OB compared to canonical theta oscillations implied that there were

155 trials in which the predominant $\mathrm{OB}$ frequency and the canonical dHC theta frequency either

156 differed or overlapped. We therefore grouped trials into two categories - trials with overlapping

$157 \mathrm{dHC}$ theta and $\mathrm{OB}$ frequency ( $\leq 1 \mathrm{~Hz}$ apart) and trials with non-overlapping $\mathrm{dHC}$ theta and $\mathrm{OB}$

158 frequency (>1 Hz apart) (Figure 4). Grouping of trials as overlapping or non-overlapping in

159 frequency was done independently for each task phase within a trial. For example, a trial could be

160 grouped as overlapping for analysis on the stem arm and as non-overlapping for analysis on the

161 return arm.

162

163 RROs were observed in the prefrontal-hippocampal network in parallel with either

164 movement-related or sensory-evoked theta oscillations

165 We first investigated the coordination between theta oscillations and RROs in trials with non-

166 overlapping frequencies. Theta oscillations are well defined as movement-related during periods

167 of running on the stem and running on return arms and as sensory-evoked during odor sampling.

168 The analysis therefore focused on these periods and excluded reward. During running on the stem

169 and return arms, LFP signals at the prefrontal and hippocampal recording sites showed two

170 detectable peaks in the $3-12 \mathrm{~Hz}$ range - one at a frequency of $\sim 8 \mathrm{~Hz}$ and another matching the

171 predominant $\mathrm{OB}$ frequency (Figure 4A and C). While being stationary during odor sampling

172 periods, LFP in the prefrontal-hippocampal network showed the same two peaks (Figure 4B and

173 C). Given that clear peaks that matched the OB frequency could be detected in $\mathrm{mPFC}$, dHC, and

174 vHC, we asked whether the peak at the OB frequency indicated coupled oscillations across all

175 brain regions. We measured coupling by computing the coherence spectra between OB oscillations

176 and prefrontal as well as hippocampal oscillations. Consistent with coupled RROs across brain 
177 regions, the maximum of the coherence spectra between the $\mathrm{OB}$ and each of the other recording

178 sites was at frequencies matching the predominant $\mathrm{OB}$ frequency. These results indicate that

179 broadly transmitted RROs were clearly detectable across brain regions and even at dorsal

180 hippocampal recording sides where canonical theta oscillations showed a substantially higher

181 amplitude than RROs (Figure 4C).

182

183 Since most trials with nonoverlapping $\mathrm{OB}$ and $\mathrm{dHC}$ frequencies were those in which the

184 predominant $\mathrm{OB}$ frequency was below $6 \mathrm{~Hz}$, we asked whether a comparison of non-overlap trials

185 with $\mathrm{OB}$ frequency $\geq 6 \mathrm{~Hz}$ would yield similar results as for those below $6 \mathrm{~Hz}$. We found that

186 coherence was significantly lower in non-overlap trials with OB frequency $\geq 6 \mathrm{~Hz}$ compared to

187 trials with $\mathrm{OB}$ frequency $<6 \mathrm{~Hz}$ for all combinations of $\mathrm{OB}$ oscillations and cortical regions and for

188 all maze segments (return arm: $n=365$ and 378 trials with $\mathrm{OB}$ frequency $<6 \mathrm{~Hz}$ and $\geq 6 \mathrm{~Hz}$, OB-

189 mPFC: $Z=-14.70, p=6.3 e-49 ; O B-d H C: Z=-15.91, p=4.9 e-57 ;$ OB-vHC: $Z=-13.16, p=1.3 e-$

190 39, Wilcoxon signed-rank test; stem: $n=447$ and 305 trials, OB-mPFC: $Z=-8.00, p=1.2 \mathrm{e}-15$;

191 OB-dHC: $\mathrm{Z}=-10.31, \mathrm{p}=6.2 \mathrm{e}-25 ;$ OB-vHC: $\mathrm{Z}=-5.99, \mathrm{p}=2.0 \mathrm{e}-9$, Wilcoxon signed-rank test;

192 odor sampling period: $n=776$ and 212 trials, OB-mPFC: $\mathrm{Z}=-11.57, \mathrm{p}=5.5 \mathrm{e}-31$; OB-dHC: $\mathrm{Z}=-$

193 10.75, $\mathrm{p}=5.4 \mathrm{e}-27 ; \mathrm{OB}-\mathrm{vHC}: \mathrm{Z}=-7.22, \mathrm{p}=5.1 \mathrm{e}-13$, Wilcoxon signed-rank test) (Figure 4 - figure

194 supplement 1). OB oscillations at high frequencies - when differing from the frequency of the 195 predominant hippocampal and prefrontal theta oscillations - therefore do not appear to couple 196 across brain regions.

198 Movement-related and sensory-evoked theta oscillations did not couple to RROs

199 While it is expected that RROs and canonical theta (either movement-related or sensory-evoked)

200 are unlikely to couple when their frequencies are non-overlapping, we considered it plausible that 
201 RROs showed coupling with either type of theta oscillation when the frequencies were matching.

202 By selecting trials in which predominant OB frequencies and hippocampal frequencies overlapped

203 (Figure 4D and E), we were able to ask whether RROs couple to canonical theta oscillations in

$204 \mathrm{mPFC}, \mathrm{dHC}$ or $\mathrm{vHC}$ regions. If theta oscillations in the prefrontal-hippocampal network were to

205 couple to RROs in this trial type, we would expect a high coherence. In contrast, we found a

206 significant decrease in coherence in trials with overlapping frequencies compared to trials with

207 non-overlapping frequencies for all combinations of OB oscillations and cortical regions and for

208 all maze segments (return arm: $n=743$ non-overlap and 464 overlap trials, OB-mPFC: $\mathrm{Z}=-10.79$,

$209 \mathrm{p}=3.7 \mathrm{e}-27 ;$ OB-dHC: $\mathrm{Z}=-9.09, \mathrm{p}=9.6 \mathrm{e}-20 ;$ OB-vHC: $\mathrm{Z}=-8.54, \mathrm{p}=1.3 \mathrm{e}-17$, Wilcoxon signed-

210 rank test; stem: $n=752$ and 455 trials, OB-mPFC: $\mathrm{Z}=-7.67, \mathrm{p}=1.7 \mathrm{e}-14 ; \mathrm{OB}-\mathrm{dHC}: \mathrm{Z}=-4.88, \mathrm{p}$

$211=1.0 \mathrm{e}-6 ; \mathrm{OB}-\mathrm{vHC}: \mathrm{Z}=-4.82, \mathrm{p}=1.4 \mathrm{e}-6$, Wilcoxon signed-rank test; odor sampling period: $n=$

212956 and 251 trials, OB-mPFC: $Z=-10.09, p=6.4 \mathrm{e}-24 ;$ OB-dHC: $Z=-7.34, p=2.1 \mathrm{e}-13$; OB-

213 vHC: $\mathrm{Z}=-6.61, \mathrm{p}=3.9 \mathrm{e}-11$, Wilcoxon signed-rank test; Figure $\mathbf{4 F}$ and $\mathbf{5}$ ). The decrease in

214 coherence in trials with overlapping compared to non-overlapping frequencies could not be

215 explained by a difference in power of these oscillations between trial types (return arm: $n=743$

216 non-overlap and 464 overlap trials, mPFC: $Z=10.73, p=1.00 ; \mathrm{dHC}: \mathrm{Z}=19.15, \mathrm{p}=1.00$; vHC:

$217 \mathrm{Z}=13.59, \mathrm{p}=1.00$, one-tailed Wilcoxon signed-rank test; stem: $n=752$ and 455 trials, mPFC: $\mathrm{Z}$

$218=8.58, \mathrm{p}=1.00 ; \mathrm{dHC}: \mathrm{Z}=21.51, \mathrm{p}=1.00 ; \mathrm{vHC}: \mathrm{Z}=14.34, \mathrm{p}=1.00$, one-tailed Wilcoxon signed-

219 rank test; odor sampling period: $n=956$ and 251 trials, $m P F C: Z=4.90, p=1.00 ; \mathrm{dHC}: Z=14.34$,

$220 \mathrm{p}=1.00 ;$ vHC: $\mathrm{Z}=8.59, \mathrm{p}=1.00$, one-tailed Wilcoxon signed-rank test). Furthermore, a

221 comparison of running velocity in trials with overlapping and non-overlapping frequencies did not

222 reveal any difference (return: $n=743$ non-overlap and 464 overlap trials, $Z=-1.44, p=.07$, stem:

$223 n=752$ and 455 trials, $\mathrm{Z}=7.21, \mathrm{p}=1.00$, one-tailed Wilcoxon signed-rank test). Taken together, 
these results suggest that either type of canonical theta oscillation - movement-related or sensory-
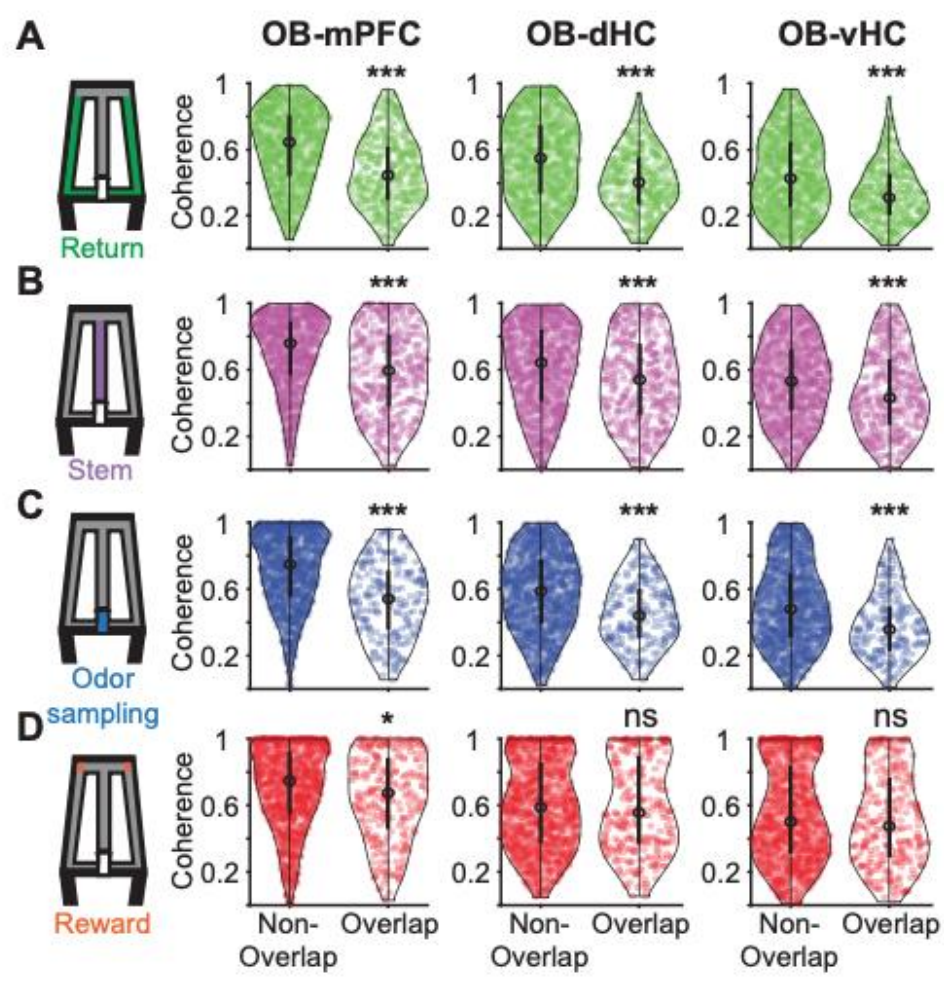

Figure 5. Respiration-entrained oscillations in the $O B$ were not coupled to movementrelated and sensory-evoked theta oscillations in the mPFC-dHC-vHC network. Violin plots of OB-mPFC, OB-dHC, and OB-vHC coherence in (A) the return arm, $(\mathbf{B})$ the stem arm, $(\mathbf{C})$ the odor sampling period and $(\mathbf{D})$ the reward arm. Coherence was calculated at the RRO frequency in each trial, and trials with overlapping and non-overlapping theta and RRO frequencies were analyzed separately. A significant decrease in coherence was found in trials with overlapping RRO and theta frequencies compared to trials with non-overlapping frequencies for all prefrontal-hippocampal regions in the return $\operatorname{arm}(n=743$ non-overlap and 464 overlap trials, OB-mPFC: $Z=-10.79, p=3.7 \mathrm{e}-27$; OB-dHC: $\mathrm{Z}=-9.09, \mathrm{p}=9.6 \mathrm{e}-20$; OBvHC: $Z=-8.54, p=1.3 e-17$, Wilcoxon signed-rank test $)$, stem arm $(n=752$ and 455 trials, OBmPFC: $Z=-7.67, p=1.7 e-14$; OB-dHC: $Z=-4.88, p=1.0 \mathrm{e}-6 ; O B-v H C: Z=-4.82, p=1.4 \mathrm{e}-6$, Wilcoxon signed-rank test $)$ and odor sampling period ( $n=956$ and 251 trials, OB-mPFC: $Z=-$ 10.09, $\mathrm{p}=6.4 \mathrm{e}-24$; OB-dHC: $\mathrm{Z}=-7.34, \mathrm{p}=2.1 \mathrm{e}-13$; OB-vHC: $\mathrm{Z}=-6.61, \mathrm{p}=3.9 \mathrm{e}-11$, Wilcoxon signed-rank test). In the reward zone, only OB-mPFC coherence ( $n=917$ and 290 trials, $Z=-$ $3.07, \mathrm{p}=0.0021$, Wilcoxon signed-rank test) was decreased in trials with overlapping RRO and theta frequencies compared to trials with non-overlapping frequencies. In the violin plots, the center circle indicates the median, and the bottom and top of the thick black lines indicate the $25^{\text {th }}$ and $75^{\text {th }}$ percentile of the data respectively. 

coherent in the stem compared to return arm regions, we explored whether we could nonetheless identify dynamic coordination of movementrelated theta oscillations between cortical areas, which has previously been described in similar tasks. We analyzed the two task phases (stem and return arms) when movement-related theta

(mPFC: $n=1207$ trials, $\mathrm{Z}=9.78, \mathrm{p}=1.00 ; \mathrm{dHC}: n=1207$ trials, $\mathrm{Z}=0.75, \mathrm{p}=0.22$, one-tailed

237 Wilcoxon signed-rank test), vHC theta power was higher in the stem compared to the return arms

238 (vHC: $n=1207$ trials, $\mathrm{Z}=-4.30, \mathrm{p}=5.82 \mathrm{e}-7$, Wilcoxon signed-rank test; Figure 6A and B).

239 Although there was a modest increase in movement-related theta power in only the vHC,

240 coherence of movement-related theta oscillations between all three pairs of regions was higher in

241 the stem compared to the return arms (mPFC-dHC: $n=1207$ trials, $\mathrm{Z}=-14.34, \mathrm{p}=4.54 \mathrm{e}-47$;

242 mPFC-vHC: $n=1207$ trials, $\mathrm{Z}=-11.49, \mathrm{p}=1.67 \mathrm{e}-29 ; \mathrm{dHC}-\mathrm{vHC}: n=1207$ trials, $\mathrm{Z}=-14.64, \mathrm{p}=$

243 3.93e-50, Wilcoxon signed-rank test; Figure 6C and D). This analysis does not only reproduce

244 previous findings, but also confirms that our methods can, in principle, detect behavior-dependent

245 increases in coherence.

Coherence between prefrontal cortex and hippocampal regions was unrelated to odor-

\section{8 guided memory performance}

249 Even though we examined odor-cued working memory in our task, the figure- 8 maze is often used 250 to assess spatial alternation behavior in rodents. While we did not train the mice to alternate in the 

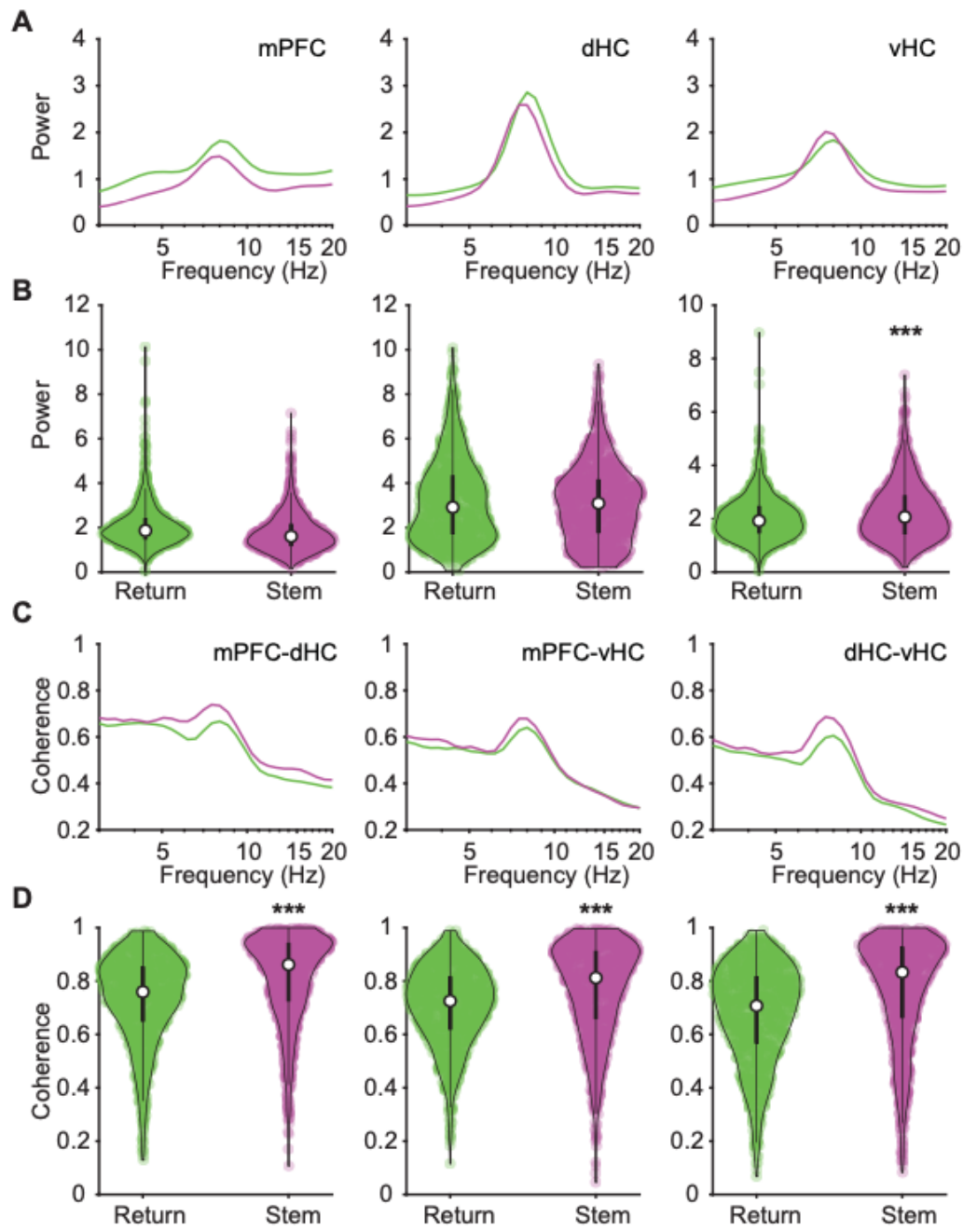

Figure 6. Movement-related theta oscillations in the prefrontal-hippocampal regions were more coherent in the stem arm compared to return arm. A. Trial-averaged power spectra of $\mathrm{mPFC}, \mathrm{dHC}$ and $\mathrm{vHC}$ regions for periods on return arms (green) and on the stem arm (purple). B. Violin plots of peak movement-related theta power in the $\mathrm{mPFC}, \mathrm{dHC}$ and vHC regions during periods on the stem and return arms across all trials. The $\mathrm{mPFC}$ and $\mathrm{dHC}$ regions did not show an increase in movement-related theta power in the stem arm compared to the return arm $(n=1207$ trials, mPFC: $\mathrm{Z}=9.78, \mathrm{p}=1.00 ; \mathrm{dHC}: \mathrm{Z}=0.75, \mathrm{p}=0.22)$, but $\mathrm{vHC}$ theta power was higher in the stem arm compared to the return arm (vHC: $\mathrm{Z}=-4.30, \mathrm{p} 5.82 \mathrm{e}-7$, onetailed Wilcoxon signed-rank test). C. Trial-averaged coherence spectra for pairs of regions (mPFC-dHC, mPFC-vHC and dHC-vHC). D. Violin plots of the coherence of movementrelated theta oscillations. Coherence of all three pairs of regions was compared between the stem (purple) and return (green) arms. Coherence of movement-related theta oscillations was higher in the stem arm compared to the return arm $(n=1207$ trials, mPFC-dHC: $Z=-14.34, p$ $=4.54 \mathrm{e}-47$; mPFC-vHC: $\mathrm{Z}=-11.49, \mathrm{p}=1.67 \mathrm{e}-29 ; \mathrm{dHC}-\mathrm{vHC}: \mathrm{Z}=-14.64, \mathrm{p}=3.93 \mathrm{e}-50$, Wilcoxon signed-rank test). In the violin plots, the center circle indicates the median, and the bottom and top of the thick black lines indicate the $25^{\text {th }}$ and $75^{\text {th }}$ percentile of the data, respectively. 
251 odor-cued task, we observed spatial alternation on successive trials (i.e., right turn followed by a

252 left turn or vice versa) in the beginning of behavioral training when the odor-cued choice behavior

253 was at chance. We reasoned that mice's propensity towards spatial alternation may continue to

254 interfere with odor-cued choices even after the mice performed above chance in the odor-cued

255 version. To test this possibility, we analyzed the four different combinations of trial types -

256 alternating correct, alternating incorrect, non-alternating correct, non-alternating incorrect - with

257 correct and incorrect referring to the odor-guided response and alternating and non-alternating

258 referring to the turn direction compared to the previous choice. Although not rewarded, alternation

259 behavior was above chance $(n=8$ mice, $\mathrm{Z}=2.28, \mathrm{p}=0.011$, one-tailed Wilcoxon signed-rank

260 test). However, there was no interaction with the odor-guided responses (correct odor-guided

261 responses in $68.8 \%$ of trials; alternation behavior in $63.8 \%$ of trials; $\left.\chi^{2}(1,1207)=0.01, p=0.92\right)$.

263 Given that we observed choices that were guided by the odor and also choices that were consistent

264 with alternation above chance, we analyzed the LFP signal that occurred immediately preceding

265 the choice point (i.e., on the stem arm) across different types of trials. Coherence between pairs of

266 regions in the mPFC-dHC-vHC network in the stem arm was not different between trials with

267 correct and incorrect odor-cued responses $(n=831$ correct and 376 incorrect trials, mPFC-dHC: $\mathrm{Z}$

$268=-0.48, \mathrm{p}=0.63 ;$ mPFC-vHC: $\mathrm{Z}=-0.05, \mathrm{p}=0.96 ; \mathrm{dHC}-\mathrm{vHC}: \mathrm{Z}=-0.77, \mathrm{p}=0.44$, Wilcoxon

269 signed-rank test; Figure 7A). However, we found that coherence was significantly higher during

270 trials with alternating choices compared to same-side (i.e., non-alternating) choices irrespective of

271 whether they were correct or incorrect with respect to the odor ( $n=771$ alternating and 436 non-

272 alternating trials, mPFC-dHC: $\mathrm{Z}=2.99, \mathrm{p}=0.003 ; \mathrm{mPFC}-\mathrm{vHC}: \mathrm{Z}=2.37, \mathrm{p}=0.02 ; \mathrm{dHC}-\mathrm{vHC}: \mathrm{Z}$

$273=2.28, p=0.02$, Wilcoxon signed-rank test; Figure 7B). These results are consistent with reports 
274 that prefrontal-hippocampal theta synchrony increases at the choice-point of a Y- or W-maze

275 during a spatial alternation tasks (Benchenane et al., 2010; Jones \& Wilson, 2005; Tavares \& Tort,

276 2021). Coherence of canonical theta in the prefrontal-hippocampal network is therefore unrelated

277 to odor-guided choices but rather seems to reflect the extent to which the mice use spatially guided

278 behavior.
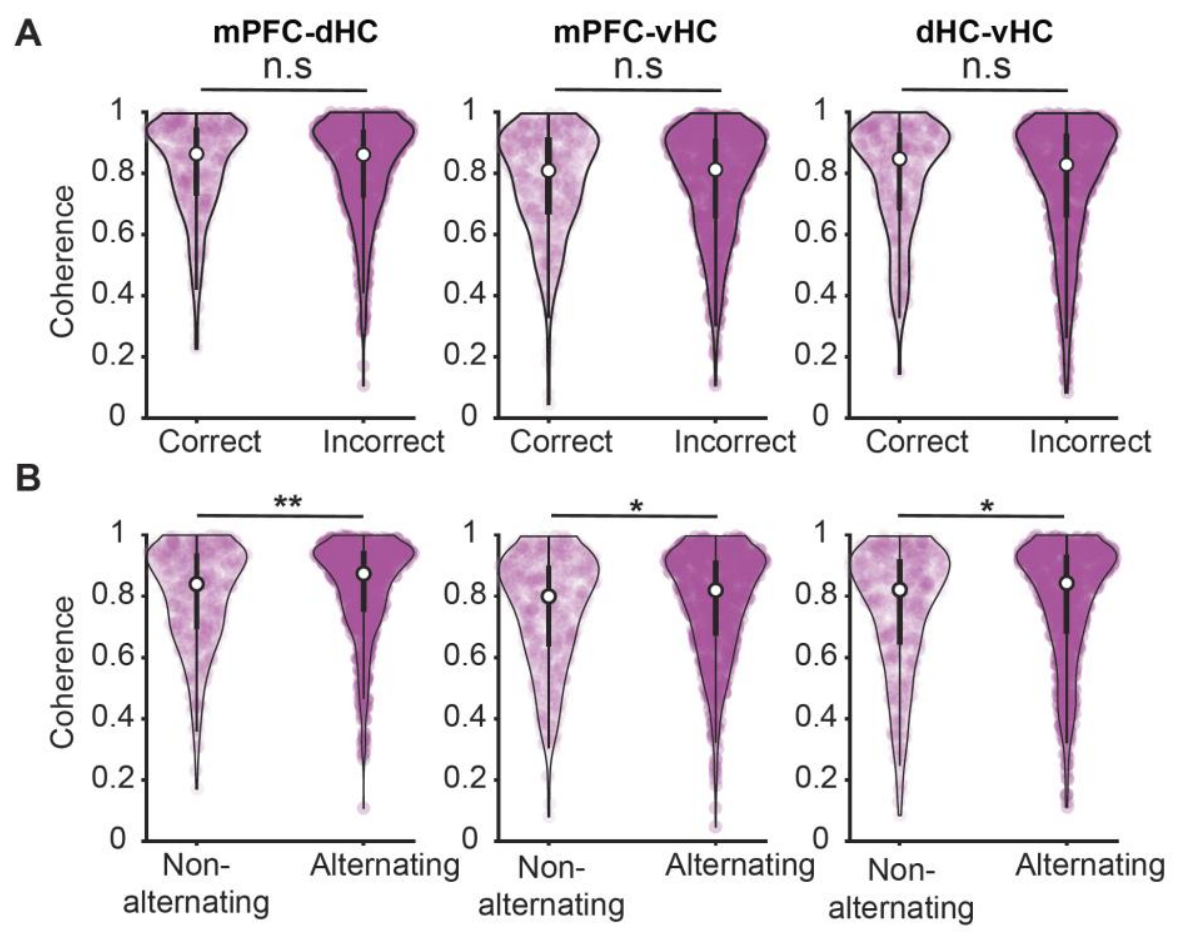

Figure 7. Movement-related theta oscillations in the stem arm were more coherent when mice alternated between left and right arms than when choosing the same arm. A. Coherence of movement-related theta oscillations between pairs of regions in the mPFC-dHCvHC network in the stem arm is compared between trials with correct and incorrect choices. Coherence was not different between trials with correct and incorrect choices $(n=831$ correct and 376 incorrect trials, mPFC-dHC: $Z=-0.48, p=0.63$; mPFC-vHC: $Z=-0.05, p=0.96$; dHCvHC: $Z=-0.77, p=0.44$, Wilcoxon signed-rank test). B. Same as A, but for alternating compared to non-alternating choices. Coherence was significantly higher during trials with alternating choices compared to trials with non-alternating choices $(n=771$ alternating and 436 nonalternating trials, mPFC-dHC: $Z=2.99, p=0.003$; mPFC-vHC: $Z=2.37, p=0.02$; dHC-vHC: $\mathrm{Z}=2.28, \mathrm{p}=0.02$, Wilcoxon signed-rank test). In the violin plots, the center circle indicates the median, and the bottom and top of the thick black lines indicate the $25^{\text {th }}$ and $75^{\text {th }}$ percentile of the data, respectively. 


\section{DISCUSSION}

281 Synchronized oscillations are thought to facilitate coordinated computations across brain regions.

282 Although it is well established that respiration-entrained oscillations are propagated from the OB

283 to other cortical areas, it is unclear to what extent these respiration-entrained oscillations get

284 coupled to endogenous theta oscillations in the prefrontal-hippocampal regions. If coupling occurs,

285 it would be an indication that oscillations that are generated by two different mechanisms in the

286 brain could dynamically couple to support memory computations. To investigate the coordination

287 of respiration-entrained oscillations in the $\mathrm{OB}$ and of theta oscillations in the prefrontal-

288 hippocampal circuit, we analyzed simultaneously recorded LFP signals from the OB, mPFC, dHC

289 and vHC during an odor-cued working memory task. We found that respiration-entrained

290 oscillations in the OB were distributed across the 3-12 Hz frequency range within each task phase,

291 including phases when animals moved and phases when animals were predominantly immobile.

292 By examining these task phases separately, we were able to test whether movement-related and

293 sensory-evoked theta oscillations in the prefrontal-hippocampal circuit couple with respiration-

294 entrained oscillations mediated by the olfactory bulb. For movement-related theta, we found that

295 OB and cortical oscillations either occurred at different frequencies, or when occurring at similar

296 frequencies, remained uncoupled from respiration-entrained oscillations in the OB. A similar result

297 was also observed for sensory-evoked theta oscillations during odor sampling periods. Taken

298 together, respiration-entrained oscillations that were propagated from the OB to prefrontal-

299 hippocampal regions did not become coupled to local theta oscillations even during odor sampling

300 periods when olfactory inputs can be assumed to strongly drive information processing and

301 sensory-evoked theta. RROs in the prefrontal-hippocampal regions therefore remained

302 independent from canonical theta oscillations and increased coupling of the oscillations did not 
appear to serve as a conduit for information processing in an odor-cued working memory task.

304 This was even observed for ventral hippocampal recording locations, which have strong

305 connections with mPFC and piriform cortex and which have been implicated to be an important

306 brain region for odor working memory (Hoover \& Vertes, 2007; Kesner et al., 2011). Rather than

307 finding a relation between odor-cued behavior and the coherence between RROs and theta, we

308 found that movement-related theta oscillations were more coherent within the prefrontal-

309 hippocampal network when the spatial component of the task guided the behavioral responses.

\section{Breathing frequency is variable but only weakly controlled by ongoing behavior}

312 It has long been known that rodent breathing frequencies can vary over a wide range. Mice have a

313 "passive" breathing frequency of 1-4 Hz during quiescence (Jessberger et al., 2016; Wesson et al.,

314 2011). Upon exposure to a novel odor, mice begin "active" sniffing at a high frequency of 4-12

315 Hz (Jessberger et al., 2016; Wesson et al., 2008; Wesson et al., 2011). Such a modulation of

316 respiration frequencies during odor sampling has been thought to be the basis for odor processing

317 in lower-order olfactory circuits (Wesson et al., 2008). Indeed, sniffing frequency changes the

318 number of odor molecules arriving at the olfactory sensory neurons, thereby increasing their

319 responsiveness to odors at these higher sniffing frequencies (Courtiol et al., 2011). However,

320 further investigations of the role of sniffing frequencies in odor information processing has

321 revealed that mice have varied strategies in terms of sniffing frequencies (Reisert et al., 2020;

322 Wesson et al., 2008). While sniffing frequencies increase in response to a novel odor sampling,

323 mice are able to perform "easy" as well as "difficult" odor discrimination tasks without a

324 significant increase in their sniffing frequencies compared to baseline (Wesson et al., 2008;

325 Wesson et al., 2009). Moreover, the relationship between sniffing frequencies and odor guided 
326 behavior is confounded by multiple factors such as locomotion and reward expectation, both of

327 which lead to increased sniffing rates (Bramble \& Carrier, 1983; Clarke, 1971; Hérent et al., 2020;

328 Wesson et al., 2008). Our results are consistent with a weak control of breathing frequencies by

329 ongoing behavior because we find that a similarly broad range of OB oscillations can occur in any

330 of the behavioral phases in an odor-cued working memory task.

\section{Coupling of movement-related theta and sensory-evoked theta to RROs}

333 Although oscillations in the $4-12 \mathrm{~Hz}$ band are broadly referred to as theta, it is well established

334 that theta oscillations in the hippocampus are of at least two types - type I and type II. Type I theta

335 is atropine-insensitive and is movement-related (Kramis et al., 1975; Vanderwolf, 1969). Power

336 and frequency of type I theta oscillations have been shown to increase with higher running speeds

337 (Feder \& Ranck, 1973; Kuo et al., 2011). Our analysis of movement-related theta oscillations in

338 the return and stem arms revealed that theta oscillations during those periods showed similar

339 relations to movement as type I theta (Figure 3 - figure supplement 1A). On the other hand,

340 type II theta is atropine sensitive and is unrelated to movement (Kramis et al., 1975; Vanderwolf,

341 1969). Type II theta is elicited when the animal is exposed to arousing, vigilant and aversive

342 conditions, such as a predator's smell (Sainsbury et al., 1987). Our recording of sensory-evoked

343 theta oscillations during the odor sampling period is akin to type II theta oscillations although we

344 did not test the atropine sensitivity of these oscillations. However, we confirmed that these theta

345 oscillations occur while the mouse's nose was held in the odor port and stationary (Figure 3A and

346 B and Figure 3 - figure supplement 1B), which suggests that theta oscillations during this task

347 phase fulfill at least one of the criteria for type II theta. By including task phases in the analysis 
when theta was either movement-related or sensory-evoked, we were therefore able to test to what extent each type of theta was related to RROs.

351 For movement-related theta oscillations in the hippocampus, seminal work that recorded nasal air

352 flow as well as LFP from the OB and dorsal hippocampus found that the hippocampal movement-

353 related theta does not couple with the respiration rhythm during exploration (Vanderwolf \&

354 Szechtman, 1987). Similarly, RROs were identified as a separate oscillation from theta oscillation

355 during running based on differences in the depth profiles across hippocampal recording sites

356 between both types of oscillations (Nguyen Chi et al., 2016). These reports correspond to our

357 finding that movement-related theta and RROs remain separate oscillations during all phases of

358 behavior. However, when investigating coupling between the two types of oscillations, Tort et al.

359 (2018) reported that theta oscillations within the hippocampus and mPFC were coherent with the

360 respiration rhythm during exploration. While it is therefore possible that RROs become the

361 predominant type of oscillation at a subset of recording sites (e.g., in dentate gyrus), we show that

362 there are also additional prefrontal and hippocampal high-amplitude theta oscillations that do not

363 couple to OB oscillations and are restricted to a more narrow frequency band than RROs.

365 The evidence for coupling between RROs and sensory-evoked theta during odor sampling is 366 stronger than for movement-related theta but also not equivocal. For example, Macrides et al. 367 (1982) reported that during odor sampling, theta oscillations in the hippocampus couple with the 368 respiration rhythm during the initial stages of learning an odor discrimination reversal task, but 369 that coherence between these oscillations was low in expert animals. Conversely, Kay (2005) 370 showed that hippocampal theta oscillations and the sniffing rhythm were coherent during odor 
371 sniffing in a two-odor discrimination task and that the coherence was positively correlated to

372 performance, which suggests that coherence remained high even when animals were proficient.

373 These discrepancies could, at least in part, be explained by the consideration that both canonical

374 theta and RROs can be recorded with hippocampal electrodes. What is interpreted as coherence

375 between hippocampal theta and respiration-entrained OB oscillations could therefore be coherence

376 between OB oscillations and RROs that can be recorded in the hippocampus. Such hippocampal

377 RROs are readily detectable in previous studies and in our data and are particularly pronounced

378 for electrodes in the dentate gyrus (Nguyen Chi et al., 2016; Yanovsky et al., 2014) and at ventral

379 hippocampal sites (see Figure 4). If we were to focus on the RRO component of our LFP signals,

380 we would indeed find that it is strongly coupled to OB oscillations during odor sampling (see

381 Figure 4C), similar to what has been reported during immobility (Nguyen Chi et al., 2016; Tort

382 et al., 2018). However, we also find that RROs are not the only type of oscillation that can be

383 recorded in hippocampus and mPFC during odor sampling. A second type of oscillation was

384 revealed that showed a narrower frequency distribution than RROs at the same recording sites and

385 corresponded to canonical theta oscillations during the odor sampling phase (see Figure 3B).

387 Our results therefore concur with existing narratives that respiration-entrained oscillations are

388 detected in the prefrontal-hippocampal areas and can be particularly evident when the respiration

389 frequency is lower than the theta oscillation frequency in the hippocampus (Nguyen Chi et al.,

390 2016). In fact, a majority of investigations have studied periods of low respiration frequency $(<6$

391 Hz) (Lockmann et al., 2016; Nguyen Chi et al., 2016; Yanovsky et al., 2014) while respiration

392 frequencies can be well above $10 \mathrm{~Hz}$ and thus be higher than the canonical theta frequency.

393 However, while mitral and tufted cells in the OB are entrained to the respiration rhythm at low 
394 frequencies (up to $6 \mathrm{~Hz}$ ), they fire tonically at higher respiratory frequencies $(6-12 \mathrm{~Hz})(\mathrm{Kay} \&$

395 Laurent, 1999). Could it therefore be the case that respiration-entrained oscillations in the OB are

396 transmitted differently to downstream cortical areas based on frequency? In the subset of trials

397 with non-overlapping RRO and theta frequencies when RRO frequency was higher than $6 \mathrm{~Hz}$,

398 respiration-entrained OB oscillations did not show increased coherence with hippocampal

399 oscillations at the RRO frequency, which differs from the pronounced coherence when RRO

400 frequency was lower than $6 \mathrm{~Hz}$. Our results are therefore consistent with those by Kay \& Laurent

401 (1999), who suggested that higher OB oscillation frequencies may not be forwarded to cortical

402 areas due to the varied entrainment of OB cells to the respiration rhythm. In addition to a lack of

403 coupling that arises from separate pacemakers - nasal air flow for RROs (Onoda \& Mori, 1980;

404 Phillips et al., 2012; Wu et al., 2017) and medial septal area for canonical theta (Bland \& Bland,

405 1986; Gaztelu \& Buño, 1982; Mitchell et al., 1982) - coupling could also be lost by the limited

406 propagation of RROs to cortical networks once the respiration rhythm as high in frequency as the

407 predominant hippocampal theta frequency.

409 Are coupled oscillators across brain regions related to behavioral performance?

410 Previous studies firmly established that RROs propagate from the OB to downstream brain regions

411 in a variety of brain states including anesthesia, mobility and immobility (Biskamp et al., 2017;

412 Fontanini et al., 2003; Ito et al., 2014; Lockmann et al., 2016; Nguyen Chi et al., 2016; Yanovsky

413 et al., 2014). Based on these observations, it was speculated that respiration-entrained oscillations

414 are a global signal that synchronizes activity across multiple brain regions and supports

415 sensorimotor integration in a context dependent manner (Lockmann et al., 2016; Macrides et al.,

416 1982; Nguyen Chi et al., 2016; Tort et al., 2018; Yanovsky et al., 2014). However, at least one 
417 previous study that tested functional coupling at the theta frequency did not find coherent

418 oscillations between the $\mathrm{OB}$ and hippocampus. Because these results were obtained in a simple

419 hippocampus-independent odor discrimination task (Fortin et al., 2002), we considered the

420 possibility that coupling between $\mathrm{OB}$ and hippocampal oscillations could emerge in a task that

421 involves the learning of associations between odors and spatial locations, which has been shown

422 to be hippocampus-dependent (Gilbert \& Kesner, 2004). However, we found that RROs were not

423 coupled to theta oscillations in either the hippocampus or mPFC during any task phase including

424 during odor sampling when the encoding of sensory information to support working memory was

425 hypothesized to most likely engage hippocampal computations. Given that we did not detect

426 coupling between respiratory-related oscillations and canonical theta oscillations, we conclude that

427 each type of oscillation supports a different set of computations and that coupling of sensory to

428 memory processing occurs in a different frequency band and/or different anatomical pathway, such

429 as by beta oscillations in the lateral entorhinal cortex (Igarashi et al., 2014). Our results therefore

430 imply that oscillatory patterns, even when co-occurring in the same brain regions at overlapping

431 frequencies in a task that engages these brain regions, can continue to support distinct

432 computations.

434 MATERIALS AND METHODS

435 Subjects

436 Eight mice (VGAT-cre 129S6(FVB)-Slc32a1 ${ }^{\mathrm{tm} 2(\mathrm{cre}) \text { Lowl }} /$ MwarJ, Jackson Labs; $n=4$ male, $n=4$

437 female) that were 4 months old and weighed 20-30 grams were used as subjects. Sample sizes

438 were determined based on the number of mice used in previous studies with recordings of RROs

439 in awake behaving mice. All mice were single-housed in a reverse $12 \mathrm{hr}$ dark/light cycle (lights 
440 off at 8 am). Mice were restricted to $85-90 \%$ of their ad libitum weight and given full access to

441 water. All the training and testing was conducted during the dark phase. All procedures were

442 conducted in accordance with the University of California, San Diego Institutional Animal Care

443 and Use Committee.

445 Surgery

446 Mice were anesthetized with isoflurane (induction: 3\%, maintenance: $1.5-2 \%$ ) and mounted in a

447 stereotaxic frame (David Kopf Instruments, Model 1900). The scalp was cleaned and retracted

448 using a midline incision and the skull was leveled between bregma and lambda. Five holes were

449 drilled in the skull to attach anchor screws. A hole was drilled above the cerebellum to place the

450 ground screw. Craniotomies were performed over four brain regions on the right hemisphere [OB:

$451+4.2 \mathrm{~mm}$ anteroposterior (A/P), $0.6 \mathrm{~mm}$ mediolateral $(\mathrm{M} / \mathrm{L}) ; \mathrm{mPFC}:+1.8-2 \mathrm{~mm} \mathrm{~A} / \mathrm{P}, 0.4 \mathrm{~mm} \mathrm{M/L}$;

$452 \mathrm{dHC}:-1.9 \mathrm{~mm} \mathrm{~A} / \mathrm{P}, 2.0 \mathrm{~mm} \mathrm{M/L}$; vHC: $-3.3 \mathrm{~mm} \mathrm{~A} / \mathrm{P}, 3.5 \mathrm{~mm} \mathrm{M} / \mathrm{L}]$ and dura was removed. Wires

453 were implanted in the four brain regions [OB: $-1.2 \mathrm{~mm}$ dorsoventral (D/V); $\mathrm{mPFC}:-1.4 \mathrm{~mm} \mathrm{D/V}$;

$454 \mathrm{dHC}:-1.8 \mathrm{~mm}$ D/V; vHC: $-3.5 \mathrm{~mm}$ D/V] to record local field potentials. The wires were threaded

455 through a circuit board with a connector, and the implant was secured with dental cement.

456 Postoperative care was administered as needed and mice were allowed to recover for a minimum

457 of 5 days.

\section{Histological procedures}

460 Mice were perfused with $0.1 \mathrm{M}$ phosphate-buffered saline (PBS) followed by 4\%

461 paraformaldehyde in PBS solution. Brains were post-fixed for 24 hours in $4 \%$ paraformaldehyde 462 and then cryoprotected in $30 \%$ sucrose solution for 2 days. Brains were then frozen and sliced into 
$46340 \mu \mathrm{m}$ coronal sections using a sliding microtome. Sections were mounted on electrostatic slides,

464 stained with cresyl violet and coverslipped with Permount (Fisher Scientific, SP15500) to visualize

465 recording locations. Slides were imaged using a virtual slide microscope (Olympus, VS120).

466

467 Olfactometer and odor delivery

468 A custom plastic odor port was machined, and two IR LEDs (transmitter and receiver) were placed 469 at the entrance of the odor port to detect nose pokes. These LEDs were connected to an Arduino 470 board (Arduino Mega 2560) which was programmed to detect nose pokes and deliver an odor 471 through a custom-made olfactometer. One of the two odors was pseudo-randomly delivered on 472 each trial, with a minimum interval of $2 \mathrm{~s}$ to prevent triggering the odor delivery twice within a 473 single trial. A custom written MATLAB script was used to deliver the odor as well as to send a 474 TTL pulse to the Neuralynx acquisition system to timestamp the odor delivery, nose poke in and 475 nose poke out. A hole was drilled at the bottom of the odor port to deliver the odor at a flow rate 476 of up to $1 \mathrm{~L} / \mathrm{min}$. Two neutral odors (ethyl acetate and isoamyl acetate) were used in the task. These 477 odors were freshly prepared daily in mineral oil (1:5 ratio by volume).

\section{Behavior}

480 Mice were trained on an odor-cued working memory task. The room was dimly lit and stable 481 environmental cues were placed. The task was performed on a figure- 8 maze that was $50 \mathrm{~cm}$ above 482 the ground, $75 \mathrm{~cm}$ long, and $50 \mathrm{~cm}$ wide with $5 \mathrm{~cm}$ wide runways. A custom-made olfactometer 483 was placed on one end of the stem arm. The maze was cleaned with $70 \%$ alcohol after each animal 484 used the maze. Animals were trained in phases. On the first day, animals were allowed to freely 485 explore the maze for 10 minutes for habituation. After habituation for one day, animals started the 
486 first phase of training. In the first phase, animals were gently guided to the odor port to break an

487 IR beam at the entrance of the odor port upon which an odor was delivered. Animals were required

488 to sniff the odor and run to the other end of the stem arm where they were forced to make the

489 correct choice. They were then rewarded with a single chocolate sprinkle (Betty Crocker Parlor

490 Perfect Chocolate Sprinkles) at the reward zone. Care was taken to place the chocolate sprinkle at

491 the reward location only after the animal made its choice. Animals performed 60 trials per day.

492 Once animals learned to nose poke in the odor port and run to the opposite end of the stem arm

493 without guidance in all 60 trials on two consecutive days, they were ready for the second phase.

494 In the second phase, animals performed the task without guidance to nose poke into the odor port

495 and were given a choice to turn in either direction at the other end of the stem arm. Responses on

496 all 60 trials were recorded and analyzed. The data from the second phase are reported in the Results

497 section.

\section{Electrophysiological recordings}

500 Local Field Potentials (LFP) were recorded using chronically implanted wires. Implanted wires

501 were connected to a head-mounted preamplifier and via a tether to a 32-channel digital data

502 acquisition system (Neuralynx, Bozeman, MT). Continuous LFP was sampled at $32000 \mathrm{~Hz}$ and

503 band-pass filtered between 0.1 and $1000 \mathrm{~Hz}$. Position data of a red and a green LED located on

504 either side of the head-mounted preamplifier were tracked by a video camera at a sampling

505 frequency of $30 \mathrm{~Hz}$ to determine the spatial location of the animals while they performed the task.

507 LFP Analysis

508 Raw LFP signals were down-sampled to $2000 \mathrm{~Hz}$ and a Morlet wavelet of width ratio $=6$ was 
used to determine the power and phase of the oscillations at $30 \log$-spaced frequencies in the 3-20

$510 \mathrm{~Hz}$ range. An average spectrogram was constructed for each maze zone in each trial. For each

511 frequency, phase differences between pairs of oscillations were calculated, and coherence in each

512 maze zone was computed as the length of the resultant vector of phase differences in that zone.

513 For analysis involving predominant frequencies within a region, a peak was detected in the 3-12

$514 \mathrm{~Hz}$ range for RROs and in the 6-12 Hz range for movement-related and sensory-evoked theta

515 oscillations. If no peak was detected, that trial was omitted from analysis for that maze zone.

\section{$517 \quad$ Statistics}

518 All statistics were performed using built-in functions in MATLAB (R2019b). Non-parametric tests

519 such as KS and Wilcoxon tests were performed. Corrections for multiple tests were performed 520 using the Holm-Bonferroni method.

522 Code availability

523 Code can be accessed at https://github.com/SunandhaSrikanth.

\section{COMPETING INTERESTS}

526 The authors declare no competing interests.

\section{ACKNOWLEDGEMENTS}

529 We thank members of the Jill Leutgeb and Stefan Leutgeb labs for valuable comments and

530 suggestions at various stages of this project. We thank Dr Cory Root for help with the custom

531 olfactometer set up. We also thank Mr. Vignesh Srinivasan for help with setting up the odor port. 
532 This research was supported by grants from the National Institute of Health (NS102915,

533 NS097772 and MH119179).

\section{REFERENCES}

Backus, A., Schoffelen, J., Szebényi, S., Hanslmayr, S., \& CF, D. (2016). Hippocampalhttps://doi.org/10.1016/j.cub.2015.12.048

Benchenane, K., Peyrache, A., Khamassi, M., Tierney, P. L., Gioanni, Y., Battaglia, F. P., \& Wiener, S. I. (2010). Coherent theta oscillations and reorganization of spike timing in the hippocampal- prefrontal network upon learning. Neuron, 66(6), 921-936.

Biskamp, J., Bartos, M., \& Sauer, J.-F. (2017). Organization of prefrontal network activity by

Bland, S. K., \& Bland, B. H. (1986). Medial septal modulation of hippocampal theta cell respiration-related oscillations. Scientific Reports, Published online: 28 March 2017; |

549 Bramble, D., \& Carrier, D. (1983). Running and breathing in mammals. Science (New York, N.Y.), 219(4582). https://doi.org/10.1126/science.6849136

551 Buzsáki, G. (2002). Theta oscillations in the hippocampus. Neuron, 33(3). https://doi.org/10.1016/s0896-6273(02)00586-x

553 Buzsáki, G., \& Draguhn, A. (2004). Neuronal Oscillations in Cortical Networks. 
Clarke, S. (1971). Sniffing and fixed-ratio behavior for sucrose and brain stimulation reward in the rat. Physiology \& behavior, 7(5). https://doi.org/10.1016/0031-9384(71)90133-8

Colgin, L. L. (2011). Oscillations and hippocampal-prefrontal synchrony. Current opinion in neurobiology, 21(3). https://doi.org/10.1016/j.conb.2011.04.006

Courtiol, E., Hegoburu, C., Litaudon, P., Garcia, S., Fourcaud-Trocmé, N., \& Buonviso, N. (2011). Individual and synergistic effects of sniffing frequency and flow rate on olfactory bulb activity [research-article]. https://doi.org/10.1152/jn.00672.2011.

Feder, R., \& Ranck, J. B. (1973). Studies on single neurons in dorsal hippocampal formation and septum in unrestrained rats. II. Hippocampal slow waves and theta cell firing during bar

Feldman, J. L., Del Negro, C. A., \& Gray, P. A. (2013). Understanding the rhythm of breathing: so near, yet so far. Annu Rev Physiol, 75, 423-452. https://doi.org/10.1146/annurevphysiol-040510-130049

572 Fortin, N. J., Agster, K. L., \& Eichenbaum, H. B. (2002). Critical role of the hippocampus in

Fontanini, A., Spano, P., \& Bower, J. M. (2003). Ketamine-xylazine-induced slow (<1.5 Hz) memory for sequences of events [OriginalPaper]. Nature Neuroscience, 5(5), 458-462.

575 Gaztelu, J. M., \& Buño, W. (1982). Septo-hippocampal relationships during EEG theta rhythm. Electroencephalogr Clin Neurophysiol, 54(4), 375-387. 
577 Gilbert, P., \& Kesner, R. (2004). Memory for objects and their locations: the role of the hippocampus in retention of object-place associations. Neurobiology of learning and memory, 81(1). https://doi.org/10.1016/s1074-7427(03)00069-8

Hoover, W., \& Vertes, R. (2007). Anatomical analysis of afferent projections to the medial prefrontal cortex in the rat. Brain structure \& function, 212(2). https://doi.org/10.1007/s00429-007-0150-4

Hyman, J. M., Zilli, E. A., Paley, A. M., \& Hasselmo, M. E. (2005). Medial prefrontal cortex cells show dynamic modulation with the hippocampal theta rhythm dependent on

Hérent, C., Diem, S., Fortin, G., \& Bouvier, J. (2020). Absent phasing of respiratory and locomotor rhythms in running mice. eLife, 9. https://doi.org/10.7554/eLife.61919

Igarashi, K. M., Lu, L., Colgin, L. L., Moser, M.-B., \& Moser, E. I. (2014). Coordination of entorhinal-hippocampal ensemble activity during associative learning. Nature, 510, 143147. https://doi.org/doi:10.1038/nature13162

594 Jessberger, J., Zhong, W., Brankačk, J., \& Draguhn, A. (2016). Olfactory Bulb Field Potentials and Respiration in Sleep-Wake States of Mice [Research Article]. Neural Plasticity, 2016. https://doi.org/https://doi.org/10.1155/2016/4570831 Interactions in a Spatial Memory Task. https://doi.org/10.1371/journal.pbio.0030402 
Kay, L. M. (2005). Theta oscillations and sensorimotor performance. Proc Natl Acad Sci U S A, 102(10), 3863-3868. https://doi.org/10.1073/pnas.0407920102

Kay, L. M., \& Laurent, G. (1999). Odor- and context-dependent modulation of mitral cell activity in behaving rats. Nat Neurosci, 2(11), 1003-1009. https://doi.org/10.1038/14801

Kesner, R. P., Hunsaker, M. R., \& Ziegler, W. (2011). The role of the dorsal and ventral hippocampus in olfactory working memory. Neurobiol Learn Mem, 96(2), 361-366. https://doi.org/10.1016/j.nlm.2011.06.011

Kuo, T. B., Li, J. Y., Chen, C. Y., \& Yang, C. C. (2011). Changes in hippocampal $\theta$ activity during initiation and maintenance of running in the rat. Neuroscience, 194, 27-35. https://doi.org/10.1016/j.neuroscience.2011.08.007 Rhythm in the Rat Hippocampus Independent of Theta and Slow Oscillations. $J$ sniffing and the limbic theta rhythm during odor discrimination reversal learning. $J$ Neurosci, 2(12), 1705-1717. impaired radial arm maze behavior in rats. J Neurosci, 2(3), 292-302. 
621 Moberly, A. H., Schreck, M., Bhattarai, J. P., Zweifel, L. S., Luo, W., \& Ma, M. (2018).

622 Olfactory inputs modulate respiration-related rhythmic activity in the prefrontal cortex

623 and freezing behavior. Nat Commun, 9(1), 1528. https://doi.org/10.1038/s41467-018-

$624 \quad \underline{03988-1}$

625 Nguyen Chi, V., Müller, C., Wolfenstetter, T., Yanovsky, Y., Draguhn, A., Tort, A. B., \&

626 Brankačk, J. (2016). Hippocampal Respiration-Driven Rhythm Distinct from Theta

627 Oscillations in Awake Mice. J Neurosci, 36(1), 162-177.

628 https://doi.org/10.1523/JNEUROSCI.2848-15.2016

629 Onoda, N., \& Mori, K. (1980). Depth distribution of temporal firing patterns in olfactory bulb related to air-intake cycles. J Neurophysiol, 44(1), 29-39.

Phillips, M. E., Sachdev, R. N., Willhite, D. C., \& Shepherd, G. M. (2012). Respiration drives network activity and modulates synaptic and circuit processing of lateral inhibition in the olfactory bulb. J Neurosci, 32(1), 85-98. https://doi.org/10.1523/JNEUROSCI.4278$\underline{11.2012}$

Reisert, J., Golden, G. J., Dibattista, M., \& Gelperin, A. (2020). Dynamics of odor sampling strategies in mice. PLoS One, 15(8), e0237756. https://doi.org/10.1371/journal.pone.0237756 rhythm follows all frequencies of diaphragmatic respiration in the freely behaving rat.

642 Sainsbury, R. S., Heynen, A., \& Montoya, C. P. (1987). Behavioral correlates of hippocampal type 2 theta in the rat. Physiol Behav, 39(4), 513-519. 
644 Siapas, A. G., Lubenov, E. V., \& Wilson, M. A. (2005). Prefrontal phase locking to hippocampal theta oscillations. Neuron, 46(1), 141-151. https://doi.org/10.1016/j.neuron.2005.02.028

Tavares, L. C. S., \& Tort, A. B. L. (2021). Hippocampal-prefrontal interactions during spatial decision-making - Tavares - - Hippocampus - Wiley Online Library.

Tort, A. B. L., Ponsel, S., Jessberger, J., Yanovsky, Y., Brankačk, J., \& Draguhn, A. (2018). Parallel detection of theta and respiration-coupled oscillations throughout the mouse brain. Sci Rep, 8(1), 6432. https://doi.org/10.1038/s41598-018-24629-z

Vanderwolf, C. H. (1969). Hippocampal electrical activity and voluntary movement in the rat. Electroencephalogr Clin Neurophysiol, 26(4), 407-418.

Vanderwolf, C. H., \& Szechtman, H. (1987). Electrophysiological correlates of stereotyped sniffing in rats injected with apomorphine. Pharmacol Biochem Behav, 26(2), 299-304.

Wesson, D. W., Donahou, T. N., Johnson, M. O., \& Wachowiak, M. (2008). Sniffing behavior of mice during performance in odor-guided tasks. Chem Senses, 33(7), 581-596.

Wesson, D. W., Varga-Wesson, A. G., Borkowski, A. H., \& Wilson, D. A. (2011). Respiratory and sniffing behaviors throughout adulthood and aging in mice. Behav Brain Res, 223(1), 99-106. https://doi.org/10.1016/j.bbr.2011.04.016 Between Sniff Frequency, Odor Discrimination, and Receptor Neuron Activation in the Rat [research-article]. https://doi.org/10.1152/jn.90981.2008. https://doi.org/10.1152/jn.90981.2008 
667 Wu, R., Liu, Y., Wang, L., Li, B., \& Xu, F. (2017). Activity Patterns Elicited by Airflow in the

668 Olfactory Bulb and Their Possible Functions. J Neurosci, 37(44), 10700-10711.

669 https://doi.org/10.1523/JNEUROSCI.2210-17.2017

670 Yanovsky, Y., Ciatipis, M., Draguhn, A., Tort, A. B., \& Brankačk, J. (2014). Slow oscillations in

671 the mouse hippocampus entrained by nasal respiration. J Neurosci, 34(17), 5949-5964.

672 https://doi.org/10.1523/JNEUROSCI.5287-13.2014

673 Zielinski, M. C., Shin, J. D., \& Jadhav, S. P. (2019). Coherent Coding of Spatial Position

674 Mediated by Theta Oscillations in the Hippocampus and Prefrontal Cortex. The Journal

675 of neuroscience : the official journal of the Society for Neuroscience, 39(23).

676 https://doi.org/10.1523/JNEUROSCI.0106-19.2019

677 


\section{SUPPLEMENTAL FIGURES}

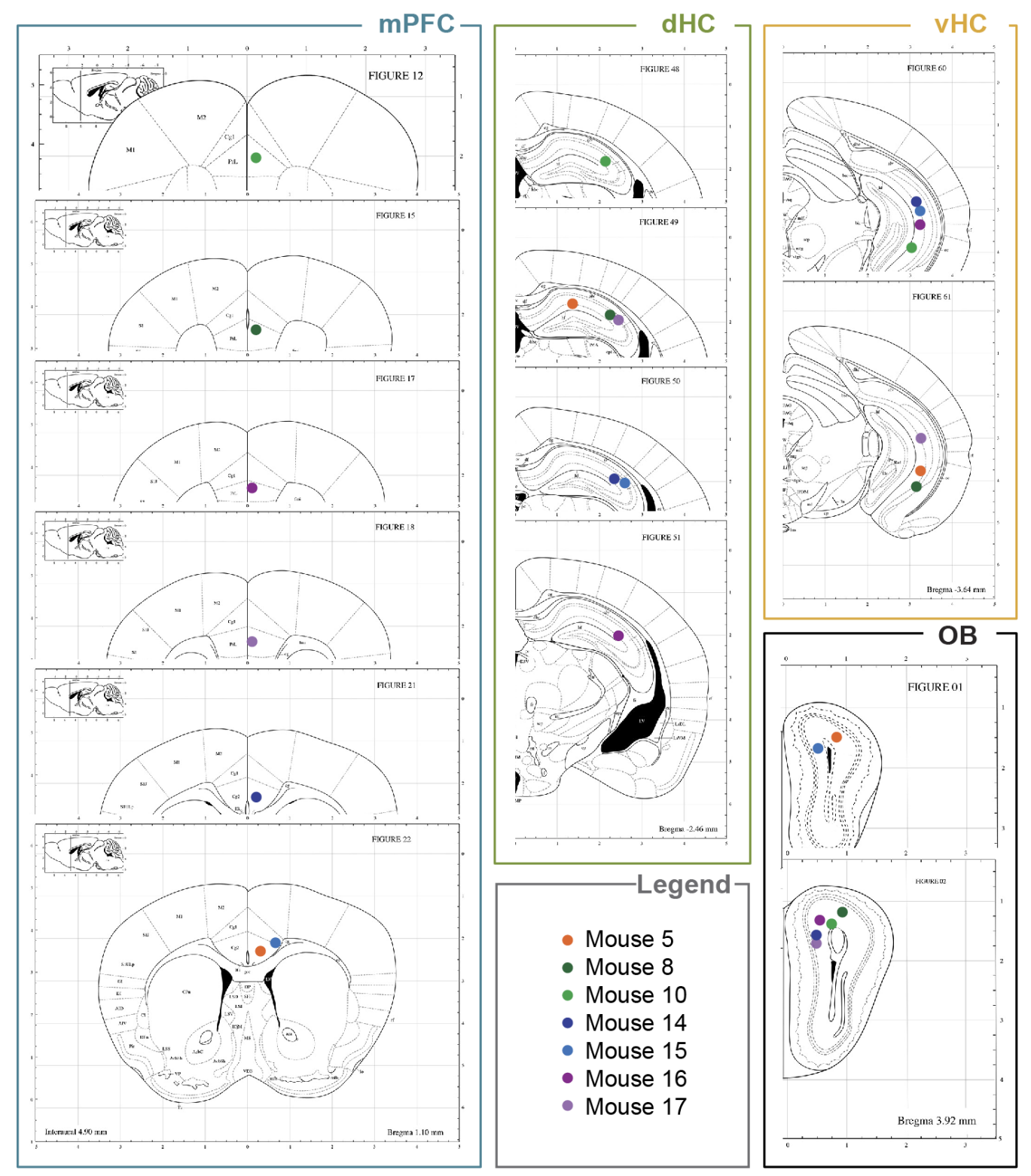

Figure 1 - figure supplement 1. Electrode recording locations. Colored dots indicate the recording locations in each animal $(n=7)$ used in the study. Electrodes were histologically confirmed to be placed in the $\mathrm{OB}$, the prelimbic, infralimbic and anterior cingulate areas of the $\mathrm{mPFC}$ and the CA1 areas of the $\mathrm{dHC}$ and the vHC. 


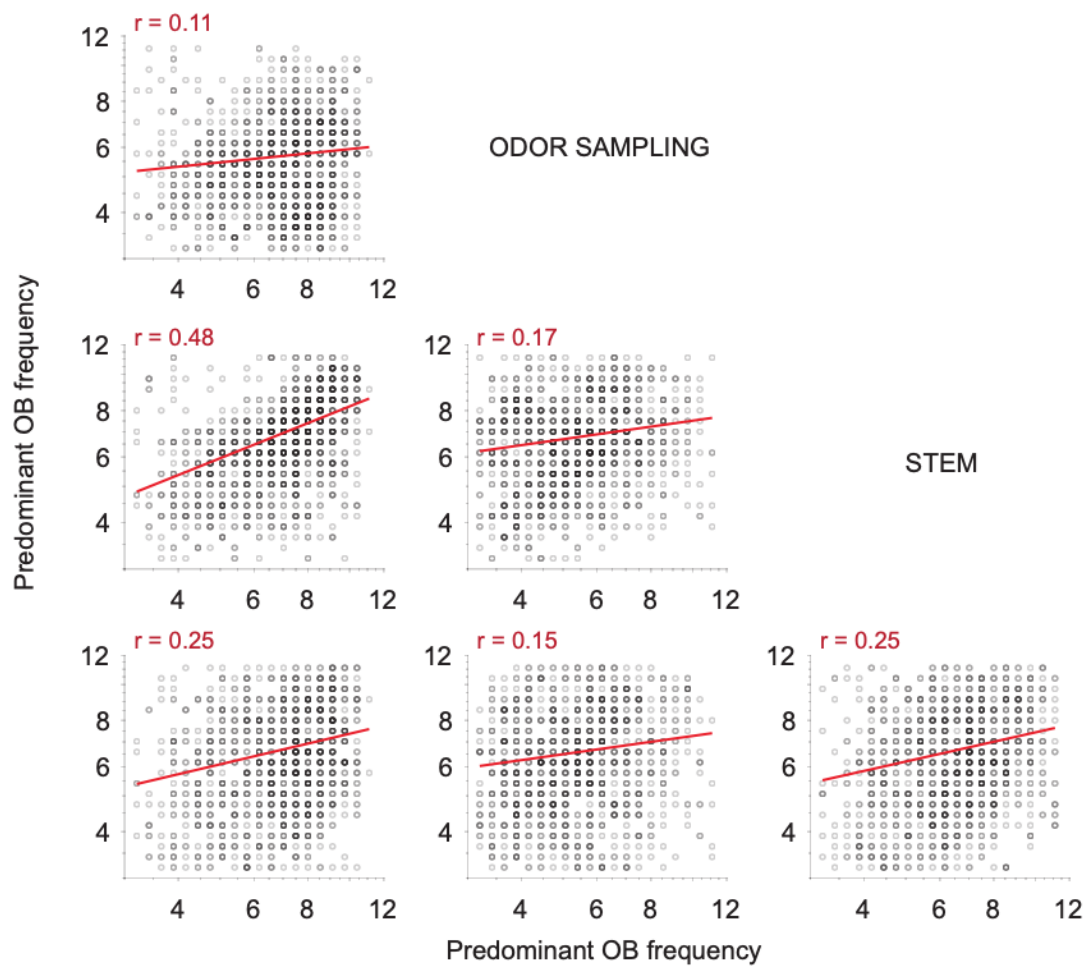

REWARD

Figure 2 - figure supplement 1 . Weak correlations between predominant $O B$ frequencies in different task phases. Scatter plots of predominant OB frequencies during different task phases in each trial. Spearman correlations between predominant OB frequencies during different task phases were weak. 
A
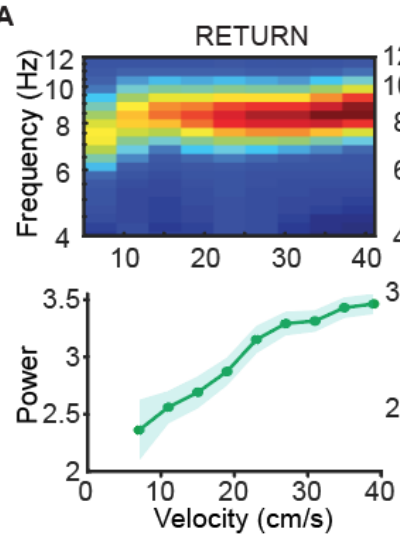

C

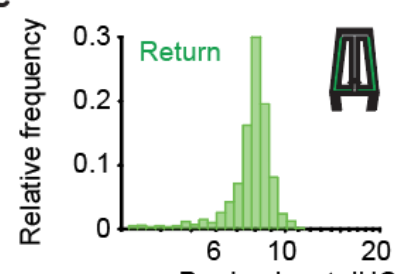

Predominant $\mathrm{dHC}$ frequency $(\mathrm{Hz})$

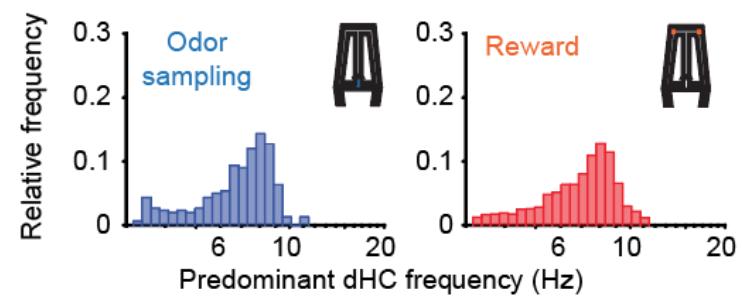

B
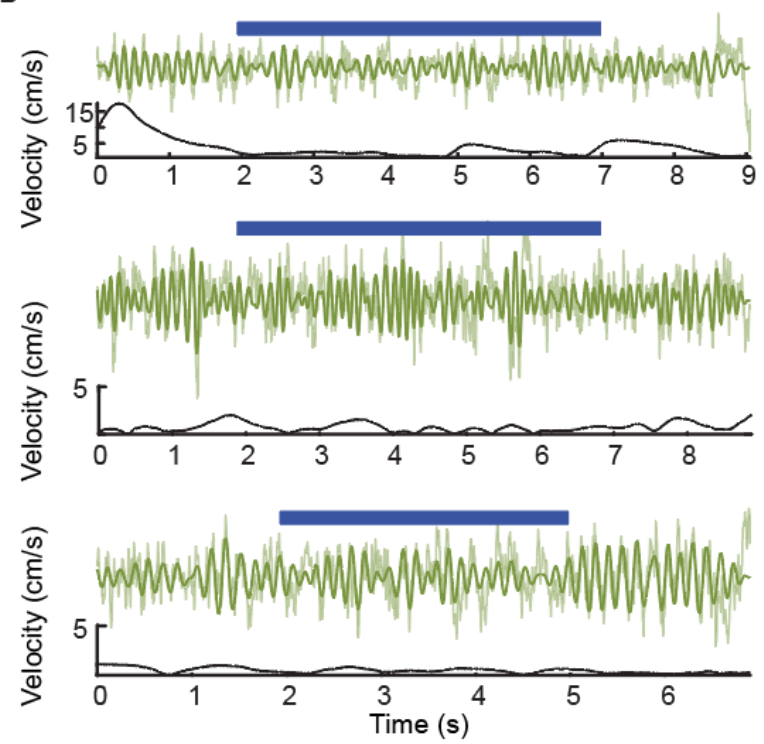

D

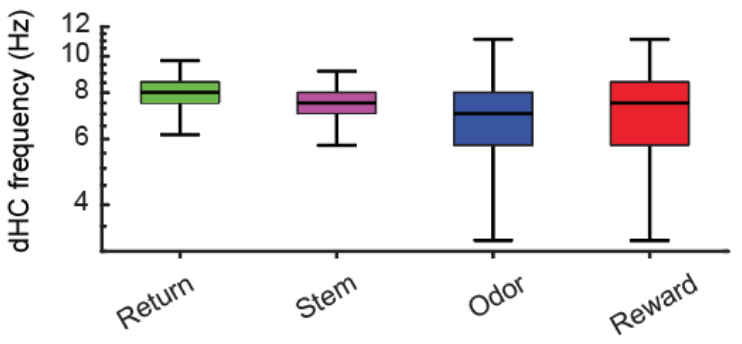

Figure 3 - figure supplement 1. Movement-related and sensory-evoked theta oscillations in the dHC. A. (Top) Mean spectrograms during periods of running on the return arms and stem arm, displaying the distribution of power and frequency of movement-related theta oscillations across running speeds. (Bottom) Corresponding average power ( \pm SEM) across running speeds. For each run through the return or the stem arm, the velocity of the animal and dHC power spectrum were calculated and the data was averaged across trials. B. Example raw (light green) and 3-12 Hz filtered (dark green) LFP traces of sensory-evoked theta oscillations in the dHC are shown in three different trials. Blue bars represent the time of odor sampling. Velocity traces accompanying each raw LFP trace show the velocity of the animal during the same time period. As expected, velocity was near zero while mice where holding their nose in the odor port. $\mathbf{C}$. Distributions of predominant dHC LFP frequencies in each task phase. D. Box plots of the predominant dHC frequency in each task phase. Data in $\mathrm{C}$ and D corresponds to Figure 3B, but more detailed distributions are shown here. In the box plots, the center line shows the median, and the bottom and top edges of the box represent the 25 th and 75 th percentiles, respectively. The whiskers indicate the most extreme data points not considered outliers. 

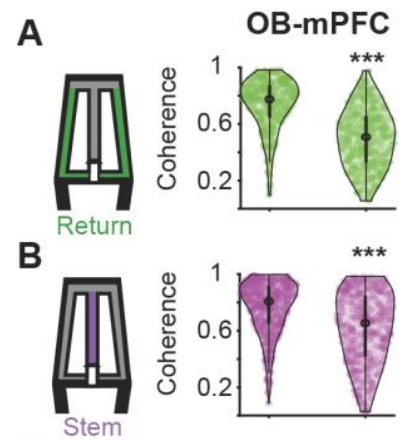

C
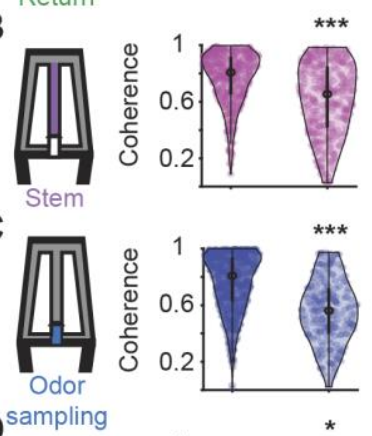

D sampling
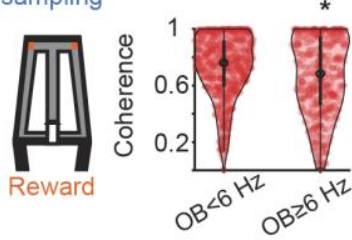
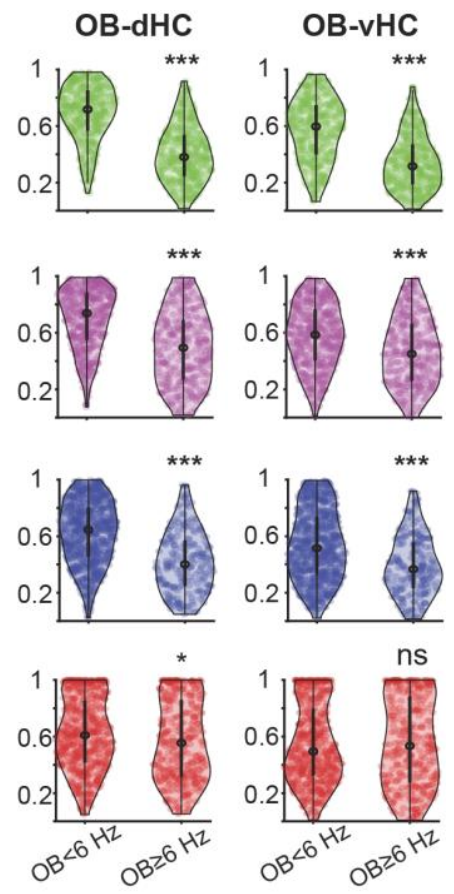

Figure 4 - figure supplement 1. Comparison of coherence between the $O B$ and the prefrontal hippocampal network in non-overlap trials with $\mathrm{OB}$ frequency $<6 \mathrm{~Hz}$ and $\geq 6 \mathrm{~Hz}$. Violin plots of OB-mPFC, OB-dHC, and OB-vHC coherence in (A) the return arm, (B) the stem arm, $(\mathbf{C})$ the odor sampling period and (D) the reward arm. Coherence was calculated at the RRO frequency in each trial. Non-overlap trials with OB frequencies $<6 \mathrm{~Hz}$ and $\geq 6 \mathrm{~Hz}$ were analyzed separately. A lower coherence was found in non-overlap trials with OB frequency $\geq 6 \mathrm{~Hz}$ compared to non-overlap trials with $\mathrm{OB}$ frequency $<6 \mathrm{~Hz}$ for all combinations of OB recordings with other brain regions in the return arm $(n=365$ and 378 trials with $\mathrm{OB}$ frequency $<6 \mathrm{~Hz}$ and $\geq 6 \mathrm{~Hz}$, OBmPFC: $Z=-14.70, p=6.3 e-49$; OB-dHC: $Z=-15.91, p=4.9 e-57 ; O B-v H C: Z=-13.16, p=1.3 e-$ 39, Wilcoxon signed-rank test), stem arm ( $n=447$ and 305 trials, OB-mPFC: $Z=-8.00, p=1.2 \mathrm{e}-$ 15; OB-dHC: $\mathrm{Z}=-10.31, \mathrm{p}=6.2 \mathrm{e}-25$; OB-vHC: $\mathrm{Z}=-5.99, \mathrm{p}=2.0 \mathrm{e}-9$, Wilcoxon signed-rank test) and odor sampling period ( $n=776$ and 212 trials, OB-mPFC: $Z=-11.57, p=5.5 \mathrm{e}-31$; OBdHC: $Z=-10.75, p=5.4 \mathrm{e}-27$; OB-vHC: $Z=-7.22, p=5.1 \mathrm{e}-13$, Wilcoxon signed-rank test). In the reward zone, only OB-mPFC ( $n=517$ and 338 trials, $Z=-2.61, \mathrm{p}=0.009$, Wilcoxon signedrank test) and OB-dHC ( $n=517$ and 338 trials, $Z=-2.64, p=0.008$, Wilcoxon signed-rank test) coherence was decreased in non-overlap trials with OB frequency $\geq 6 \mathrm{~Hz}$ compared to $<6 \mathrm{~Hz}$. In the violin plots, the center circle indicates the median, and the bottom and top of the thick black lines indicate the $25^{\text {th }}$ and $75^{\text {th }}$ percentile of the data, respectively. 